\title{
RATE OF CONVERGENCE OF THE CONFIGURATION INTERACTION MODEL FOR THE HELIUM GROUND STATE*
}

\author{
BENJAMIN D. GODDARD ${ }^{\dagger}$
}

\begin{abstract}
The rate of convergence of a CI calculation on the ground state of the helium atom is rigorously derived under suitable assumptions on the regularity of the exact wavefunction. For bases consisting of all partial waves with angular momentum less than or equal to $L$, the large $L$ asymptotic energies are found to obey the well-known formula $E_{L}-E=C L^{-3}+o\left(L^{-3}\right)$, where $C$ is an explicit constant defined in terms of the exact wavefunction.
\end{abstract}

Key words. asymptotic analysis, partial wave expansion, Schrödinger equation

AMS subject classifications. 41A25, 81Q05, 81V45

DOI. $10.1137 / 080727956$

\section{Introduction.}

1.1. History and discussion of the problem. One of the principal aims of atomic and molecular quantum chemistry is to solve the time-independent Schrödinger equation [22]

$$
H \psi=E \psi,
$$

where, in the Born-Oppenheimer [2] (infinite nuclear mass limit) nonrelativistic approximation for an atomic system with $N$ electrons and nuclear charge $Z$,

$$
H:=\sum_{i=1}^{N}-\frac{1}{2} \Delta_{\mathbf{r}_{i}}-\frac{Z}{\left|\mathbf{r}_{i}\right|}+\sum_{1 \leq i<j \leq N} \frac{1}{\left|\mathbf{r}_{i}-\mathbf{r}_{j}\right|},
$$

where the positions of the electrons are denoted by $\mathbf{r}_{i}$, and $H$ is in atomic units. $\psi$ is known as the wavefunction and $E$ the corresponding energy. Unfortunately, except in the simplest case $N=1,(1.1)$ is not exactly soluble, and thus the focus turns to finding accurate approximate solutions.

Perhaps the simplest approximation is to replace the electron-electron repulsion term $\sum 1 /\left|\mathbf{r}_{i}-\mathbf{r}_{j}\right|$ by an "average" repulsion, leading to the Hartree-Fock $[10,6,24]$ model. The energy is then minimized over all antisymmetrized products of $N$ oneelectron orbitals (functions $\varphi: \mathbb{R}^{3} \times \mathbb{Z}_{2} \rightarrow \mathbb{C}$, taking as inputs the position and spin of a single electron). These products are known as Slater determinants. However, ignoring the exact correlation leads to poor results for most systems of interest.

In more complicated quantum chemistry models, the wavefunction is expanded as a linear combination of Slater determinants. The major limitation is the slow convergence of this wavefunction expansion, and thus also the energy. This results primarily from the cusp behavior of the exact wavefunction at singularities of the Coulomb potential, i.e., at points at which two or more interparticle distances are zero, and in particular from the inability of the one-electron orbitals to accurately model these cusps $[24,14]$.

* Received by the editors June 20, 2008; accepted for publication (in revised form) January 13, 2009; published electronically April 29, 2009. This work was partly supported by the EPSRC.

http://www.siam.org/journals/sima/41-1/72795.html

$\dagger$ Mathematics Institute, University of Warwick, Coventry CV4, United Kingdom (bdgoddard@ gmail.com). 
Although it is possible to determine highly accurate energies, at least for very small atoms (see, e.g., [18] and references therein), this is much less practical for even moderately sized atoms $(N \geq 5)[9]$. The slow rate of convergence, along with the high cost of such calculations with respect to basis size (the number of $N$-electron wavefunctions grows exponentially with the number of one-electron orbitals), makes it highly desirable to derive an extrapolation scheme for the energy, allowing accurate theoretical predictions with low computational cost (see, for example, the recent work [3]). For this application it is necessary not only to derive the leading order of the error, but also the corresponding leading coefficient.

Furthermore, and perhaps most importantly, the question of the rate of convergence is of fundamental theoretical interest, and an analysis of such errors may suggest areas of improvement for the corresponding model.

The configuration interaction (CI) (see, e.g., [24]) model minimizes the energy over the span of a fixed set of Slater determinants. For computational reasons this set is taken to be finite, although theoretical formulations utilize infinite sets. Mathematically this is analogous to a Ritz-Galerkin method on the space spanned by the Slater determinants, and the analysis of the energy error is equivalent to analysis of the error of the corresponding Rayleigh-Ritz calculation.

Historically, the spaces of interest have been the subspaces $V_{L}$ of wavefunctions with angular momentum less than or equal to $L$, and the analysis has been predominantly limited to two-electron atomic and ionic systems. The radial parts of these subspaces are infinite dimensional, and hence this does not correspond to a computationally feasible CI calculation. However, the radial part is easily well-approximated (see below) and the main error results from the angular-momentum truncation. Expanding the angular component of the wavefunction, the $\ell$ th term in the partial wave expansion of a two-electron wavefunction with electron positions $\mathbf{r}_{1}$ and $\mathbf{r}_{2}$,

$$
\psi\left(\mathbf{r}_{1}, \mathbf{r}_{2}\right)=\sum_{\ell=0}^{\infty} \psi_{\ell}\left(r_{1}, r_{2}\right) P_{\ell}(\cos \theta)
$$

where $r_{i}=\left|\mathbf{r}_{i}\right|, \theta$ is the planar angle between $\mathbf{r}_{\mathbf{1}}$ and $\mathbf{r}_{\mathbf{2}}$, and $P_{\ell}(x)$ are the Legendre polynomials. This is equivalent to the contribution of one-electron functions with angular quantum number $\ell$ in an infinite-dimensional CI expansion of $\psi$, which can be seen by rewriting [15]

$$
\psi_{\ell}\left(r_{1}, r_{2}\right) P_{\ell}(\cos \theta)=\frac{4 \pi}{2 \ell+1} \sum_{i, j} c_{i j} \phi_{\ell, i}\left(r_{1}\right) \phi_{\ell, j}\left(r_{2}\right) \sum_{m=-\ell}^{\ell} Y_{\ell}^{m}\left(\theta_{1}, \phi_{1}\right) Y_{\ell}^{m *}\left(\theta_{2}, \phi_{2}\right),
$$

where $Y_{\ell}^{m}$ are the spherical harmonics. It is notable that this expansion is also slowly convergent and, due to the increasing number of terms, more slowly convergent as $\ell$ increases. While its rate of convergence has been discussed $[17,3,5]$, there are no rigorous results, which, when combined with the results of this paper, would give a rate of convergence in terms of one-electron orbitals. We note however that the expansion of the radial part in $r_{<}:=\min \left\{r_{1}, r_{2}\right\}$ and $r_{>}:=\max \left\{r_{1}, r_{2}\right\}$ converges rapidly $[4,21]$.

The first such analysis for classical orthogonal basis functions in the perturbation theoretic problem was given by Schwartz [23], who found for the $1 / Z$ perturbation theory expansion that

$$
E_{L}-E_{L-1}=-\frac{45}{256}(L+1 / 2)^{-4}+\frac{225}{1024}(L+1 / 2)^{-6}+\mathcal{O}\left((L+1 / 2)^{-8}\right),
$$


where $E_{L}$ denotes the energy obtained by including all angular momenta less than or equal to $L$. A heuristic argument that the asymptotic formula $E_{L}-E_{L-1} \sim$ $(L+1 / 2)^{-4}$ should hold for the partial wave expansion (and hence for the CI model) was given by Lakin [16]. This behavior was demonstrated numerically for the ground state of helium by Carroll, Silverstone, and Metzger [5].

The first attempt at a rigorous mathematical proof of such a result for a CI calculation was given by Hill [11] who built upon work by Klahn and Morgan [14], and claimed that $E_{L}-E=C_{1}(L+1 / 2)^{-3}+C_{2}(L+1 / 2)^{-4}+\mathcal{O}\left(L^{-5}\right)$ where $C_{1}$ and $C_{2}$ are given explicitly in terms of the exact ground state wavefunction. Note that this agrees with the asymptotic result $E_{L}-E_{L-1}=3 C_{1}(L+1 / 2)^{-4}$. However, not only is the proof of this result very difficult to follow, especially that of the $L^{-4}$ term, but it contains a number of typographical mistakes and is not entirely mathematically rigorous. For this reason we present a new proof, the main differences of which will be outlined below.

The above results hold only for the ground state of two-electron systems (i.e., He-like ions), but were extended to all states of two-electron systems for the $1 / Z$ perturbation theoretic model by Kutzelnigg and Morgan [15]. However, such extensions for CI-like methods seem more difficult.

Apart from the increased rigor, the principle differences from Hill's method concern the formulation of the expression for the energy error (Hill's (3.22)), which result in the need to analyze derivatives in $r_{<}$and $r_{>}$of $\psi_{\ell}$ rather than $\ell$-projections of $r_{12}^{-1} \psi\left(r_{1}, r_{2}, r_{12}\right)$, where $r_{12}:=\left|\mathbf{r}_{1}-\mathbf{r}_{2}\right|$. A further difference in our analysis is that we construct an explicit form of $f_{\ell}^{k}\left(r_{<}, r_{>}\right)$, the $\ell$-projections of $r_{12}^{2 k-1}$, whereas Hill uses a recursion formula to obtain an asymptotic result; this is necessary for the analysis of the derivatives of $f_{\ell}^{k}\left(r_{<}, r_{>}\right)$in $r_{<}$and $r_{>}$. The analysis of the remainder terms $R_{j, \ell}\left(r_{<}, r_{>}\right)$is similar to that of Hill but we produce a more detailed version of his Appendix C, the necessary conditions seem optimal in this formulation of the problem.

One noteworthy point is that, in order to obtain the resulting asymptotic behavior, the wavefunction is required to be three times differentiable in $r_{12}$. The validity of this assumption will be discussed in section 3 .

1.2. Overview of the paper. In section 2 we formulate the problem and introduce the necessary basic notation. In section 3 we state our main result, the proof of which is outlined in section 4 . This section also contains a statement of two, more general, results required for the proof (Theorems 4.2 and 4.3). In section 5 we prove the main result using these two theorems, along with a number of preliminary lemmas, given in section 5.1. The remainder of the paper, section 6 , is concerned with the proofs of Theorems 4.2 and 4.3, which are outlined at the start of the section.

2. Preliminaries and notation. Let $\psi$ be the actual ground state solution to the Schrödinger equation for the Helium atom, $H \psi=E \psi$ where

$$
H:=-\frac{1}{2} \Delta_{1}-\frac{1}{2} \Delta_{2}-\frac{2}{\left|\mathbf{r}_{\mathbf{1}}\right|}-\frac{2}{\left|\mathbf{r}_{\mathbf{2}}\right|}+\frac{1}{\left|\mathbf{r}_{\mathbf{1}}-\mathbf{r}_{\mathbf{2}}\right|},
$$

where $\mathbf{r}_{1}, \mathbf{r}_{2} \in \mathbb{R}^{3}$ are the positions of the two electrons and we later use the notation $\left|\mathbf{r}_{1}-\mathbf{r}_{2}\right|=: r_{12}$.

The ground state of the Helium atom has angular momentum zero (see for example the experimental data of [19]) and is therefore invariant under simultaneous rotation of $\mathbf{r}_{1}$ and $\mathbf{r}_{2}$. Hence the ground state may be described by the lengths of the two 
vectors $\mathbf{r}_{1}$ and $\mathbf{r}_{2}$ and the angle between them, denoted, respectively, $r_{1}, r_{2}$, and $\theta$. Equivalently, $\theta$ may be written in terms of $r_{1}, r_{2}$, and $r_{12}$, giving $\psi=\psi\left(r_{<}, r_{>}, r_{12}\right)$ where

$$
r_{<}:=\min \left\{r_{1}, r_{2}\right\}, \quad r_{>}:=\max \left\{r_{1}, r_{2}\right\}
$$

and

$$
r_{12}:=\left|\mathbf{r}_{1}-\mathbf{r}_{2}\right|=\left(r_{<}^{2}+r_{>}^{2}-2 r_{<} r_{>} \cos \theta\right)^{1 / 2} .
$$

We wish to investigate the energy of the approximate ground state wavefunction given by projection of $\psi$ onto the space spanned by functions with angular momentum less than or equal to $L$. It is well known [1] that the Legendre polynomials

$$
P_{\ell}(x):=\frac{1}{2^{\ell} \ell !} \frac{d^{\ell}}{d x^{\ell}}\left(x^{2}-1\right)^{\ell}
$$

are orthogonal with respect to the weighted inner product on $L^{2}$ given by

$$
(f, g):=\int_{0}^{\pi} f(\theta) \overline{g(\theta)} \sin \theta d \theta .
$$

It follows from the normalization formula [1]

$$
\int_{0}^{\pi} P_{m}(\cos \theta) P_{n}(\cos \theta) \sin \theta d \theta=\delta_{m, n} \frac{2}{2 n+1}
$$

that the corresponding orthonormal wavefunctions are given by

$$
\Phi_{\ell}(\theta)(x):=\left(l+\frac{1}{2}\right)^{1 / 2} P_{\ell}(\cos \theta),
$$

which form a complete basis under the inner product $(2.3)$. These $\Phi_{\ell}(\theta)$ are eigenfunctions of the angular momentum operator with eigenvalues $-\ell(\ell+1)$ and we are interested in the projection onto the span of the first $L$ of these, i.e.,

$$
P_{L}:=\sum_{\ell=1}^{L}\left(\cdot, \Phi_{\ell}\right) \Phi_{\ell} .
$$

We denote the expansion coefficients by $\psi_{\ell}\left(r_{<}, r_{>}\right)$:

$$
\psi_{\ell}\left(r_{<}, r_{>}\right)=\int_{0}^{\pi} \psi\left(r_{<}, r_{>}, r_{12}\right) \Phi_{\ell}(\theta) \sin \theta d \theta,
$$

and hence have

$$
P_{L} \psi\left(r_{<}, r_{>}, r_{12}\right)=\sum_{\ell=0}^{L} \psi_{\ell}\left(r_{<}, r_{>}\right) \Phi_{\ell}(\theta) .
$$

By the completeness of the $\Phi_{\ell}(\theta)$ we denote the part of the wavefunction whose angular part does not lie in the span of the first $L$ Legendre polynomials by

$$
\psi_{L}^{\perp}:=\psi-P_{L} \psi
$$

with an analogous expansion:

$$
\psi_{L}^{\perp}\left(r_{<}, r_{>}, r_{12}\right)=\sum_{\ell=L+1}^{\infty} \psi_{\ell}\left(r_{<}, r_{>}\right) \Phi_{\ell}(\theta) .
$$

Copyright $@$ by SIAM. Unauthorized reproduction of this article is prohibited. 
We now wish to investigate the error in the energy in terms of $\psi_{L}^{\perp}$. In particular, for the space

$$
S_{L}:=\operatorname{Span}\left\{\Phi_{\ell}(\theta), \ell=0, \ldots, L\right\}
$$

we are interested in the quantity

$$
E_{L}:=\inf _{\phi \in S_{L} \backslash\{0\}} \frac{\langle\phi, H \phi\rangle}{\|\phi\|^{2}} .
$$

Throughout the paper, we denote the standard $L^{2}$ norm of a function $\psi \in L^{2}$ by $\|\psi\|$ and, for functions in $\psi \in H^{1}$, we denote the "energy norm," which is simply the $H^{1}$ norm, by $\|\psi\|_{e}:=\|\nabla \psi\|$.

Finally, since the major assumptions on the wavefunction are with regards to its regularity in $r_{12}$, we find it convenient to define, for sufficiently regular functions, the explicit functions

$$
\xi_{\ell}^{j}\left(r_{<}, r_{>}\right):=\int_{0}^{\pi} \frac{\left(r_{12}-\left(r_{>}-r_{<}\right)\right)^{j}}{j !} \Phi_{\ell}(\theta) \sin \theta d \theta
$$

and coefficients

$$
A_{j}\left(r_{<}, r_{>}\right):=\left[\frac{\partial^{j}}{\partial r_{12}^{j}} \psi\left(r_{<}, r_{>}, r_{12}\right)\right]_{r_{12}=r_{>}-r_{<}}, j=0, \ldots, J-1,
$$

as well as a "remainder term"

$$
R_{J}\left(r_{<}, r_{>}, r_{12}\right):=\int_{r_{>}-r_{<}}^{r_{12}} \frac{\left(r_{12}-t\right)^{J}}{J !} \frac{\partial^{J+1}}{\partial r_{12}^{J+1}} \psi\left(r_{<}, r_{>}, t\right) d t,
$$

and its projection onto $\Phi_{\ell}(\theta)$ as

$$
R_{J, \ell}\left(r_{<}, r_{>}\right):=\int_{0}^{\pi} R_{J}\left(r_{<}, r_{>}, r_{12}\right) \Phi_{\ell}(\theta) \sin \theta d \theta .
$$

As will be seen in Lemma 5.1, these definitions are motivated by the Taylor expansion of the wavefunction.

We also find it convenient to define

$$
I_{j}\left(r_{<}, r_{>}\right):=\int_{r_{>}-r_{<}}^{r_{<}+r_{>}}\left|\frac{\partial^{j}}{\partial r_{12}^{j}} \psi\left(r_{<}, r_{>}, t\right)\right|^{2} d t
$$

and norms on the set of symmetric functions $f: \mathbb{R}_{+} \times \mathbb{R}_{+} \rightarrow \mathbb{C}$ by

$$
\|f\|_{a, b}:=\left(\int_{0}^{\infty} \int_{0}^{r}\left|f\left(r_{<}, r_{>}\right)\right|^{2} r_{<}^{a} r_{>}^{b} d r_{<} d r_{>}\right)^{1 / 2} .
$$

\section{Statement of the result.}

THEOREM 3.1. Let $\psi$ be the ground state solution of the helium atom Schrödinger equation $H \psi=E \psi$, with $H$ given by (2.1), and $\psi_{L}^{\perp}$ be as in (2.6). Suppose that $\frac{\partial^{j}}{\partial r_{12}^{j}} \psi\left(r_{<}, r_{>}, r_{12}\right)$ exist for $r_{>}-r_{<} \leq r_{12} \leq r_{<}+r_{>}, j=0, \ldots, 4$. Let $\mathcal{A}:=$ 
$\left\{A_{j}\left(r_{<}, r_{>}\right), \frac{\partial}{\partial r_{\gamma}} A_{j}\left(r_{<}, r_{>}\right), j=0, \ldots, 4, r_{\gamma} \in\left\{r_{<}, r_{>}\right\}\right\}$and suppose that all $A \in \mathcal{A}$ satisfy the condition $A\left(\cdot, r_{>}\right) \in C^{N}\left(\mathbb{R}^{+}\right)$, and there exists $\tilde{A}: \mathbb{R}^{+} \rightarrow \mathbb{R}$ such that

$$
\tilde{A}\left(r_{>}\right) \geq\left|\left[\frac{\partial^{n}}{\partial r_{<}^{n}}\left|A\left(r_{<}, r_{>}\right)\right|^{2}\right]_{r_{<}=s}\right|, \forall s \in \mathbb{R}^{+}, n \in\{0,1\}, A \in \mathcal{A} .
$$

Suppose, further, that

$$
\int_{0}^{\infty} r^{5}|\psi(r, r, 0)|^{2} d r<\infty, \text { and } \int_{0}^{\infty} r^{7}|\psi(r, r, 0)|^{2} d r<\infty
$$

and, for $I_{j}$ as in (2.12), $I_{3}\left(r_{<}, r_{>}\right), I_{4}\left(r_{<}, r_{>}\right)<\infty$ for a.e. $0 \leq r_{<} \leq r_{>}$, and

$$
\int_{0}^{\infty} \int_{0}^{r_{>}}\left(r_{<}^{3} I_{3}\left(r_{<}, r_{>}\right)+r_{<}^{7} I_{4}\left(r_{<}, r_{>}\right)\right) r_{<}^{2} r_{>}^{2} d r_{<} d r_{>}<\infty
$$

Then

$$
\begin{aligned}
\left\|\psi_{L}^{\perp}\right\|^{2} & =5 \pi^{2} \sum_{\ell=L+1}^{\infty}\left(\ell^{-6} \int_{0}^{\infty} r^{7}|\psi(r, r, 0)|^{2} d r+\mathcal{O}\left(\ell^{-7}\right)\right) \\
\left\|\psi_{L}^{\perp}\right\|_{e}^{2} & =6 \pi^{2} \sum_{\ell=L+1}^{\infty}\left(\ell^{-4} \int_{0}^{\infty} r^{5}|\psi(r, r, 0)|^{2} d r+o\left(\ell^{-4}\right)\right), \text { and } \\
E_{L}-E & =2 \pi^{2} L^{-3} \int_{0}^{\infty} r^{5}|\psi(r, r, 0)|^{2} d r+o\left(L^{-3}\right)
\end{aligned}
$$

Before we prove the result we note that the existence of the fourth derivative is only necessary for the decay of $\left\|\psi_{L}^{\perp}\right\|^{2}$ given in (3.1), and in particular it is required so that the remainder term decays faster than the first term in the expansion. If we are only interested in the error in the energy, then the existence of the third derivative is sufficient to show (3.2).

The validity of this assumption has, as far as we know, not been investigated mathematically beyond the results of [7]. This result shows that electronic wave functions $\psi$ of atoms and molecules have a representation $\psi=\mathcal{F} \phi$, where $\mathcal{F}$ is an explicit universal factor, locally Lipschitz, and independent of the eigenvalue and the solution $\psi$ itself, and $\phi$ has second derivatives which are locally in $L^{\infty}$. This representation is shown to be sharp in the case of Hydrogen, where the solutions to (1.1) are known explicitly. However, there are no known sharpness results concerning atoms with more than one electron, for which (1.1) is not exactly soluble. Hence we proceed with the proof of the theorem, making the relevant assumptions. Due to the complexity of the proof of [7], and the lack of similar results, one would assume that it is very difficult to prove such a result mathematically.

In addition, it is possible that the decay rate is the same even if the wavefunction is less regular, although a significantly different method of proof would be required. The similar results of [23] and [15] (both of which use a perturbation expansion in $1 / Z$ ), along with the numerical simulations such as [3], suggest that the derived decay rate is, indeed, correct.

4. Outline of the proof. The foundation of the proof of Theorem 3.1 is the following lemma, which is a special case of a result by Friesecke [8]. 
Lemma 4.1. Let $E$ be the lowest eigenvalue of $H$ given in (2.1). For $\psi_{L}^{\perp}$ and $E_{L}$ as in (2.6) and (2.7) the following bound on the energy error holds:

$$
0 \leq E_{L}-E \leq\left\|\psi_{L}^{\perp}\right\|_{e}^{2}+C_{1}\left\|\psi_{L}^{\perp}\right\|\left\|\psi_{L}^{\perp}\right\|_{e}+C_{2}\left(\left\|\psi_{L}^{\perp}\right\|^{2}+\left\|\psi_{L}^{\perp}\right\|_{e}^{4}\right),
$$

for some $C_{1}, C_{2}>0$.

The proof of Theorem 3.1 proceeds in a number of stages. First we Taylor expand a suitably regular wavefunction (Lemma 5.1). We then expand the norms $\left\|\psi_{L}^{\perp}\right\|^{2}$ and $\left\|\psi_{L}^{\perp}\right\|_{e}^{2}$ of Theorem 3.1 in terms of $\left\|\psi_{\ell}\left(r_{<}, r_{>}\right)\right\|_{a, b}$ and $\left\|\frac{\partial}{\partial r_{\gamma}} \psi_{\ell}\left(r_{<}, r_{>}\right)\right\|_{a, b}$ (Lemma 5.2). We then estimate these norms in the same norms of products of $A_{j}\left(r_{<}, r_{>}\right)$and $\xi_{\ell}^{j}\left(r_{<}, r_{>}\right)$and their derivatives (Lemma 5.3). The necessary decay estimates of these norms are then given by the following two theorems.

THEOREM 4.2. Let $\psi\left(r_{<}, r_{>}, r_{12}\right)$ be a function such that $\frac{\partial^{j}}{\partial r_{12}^{j}} \psi\left(r_{<}, r_{>}, r_{12}\right)$ exist for $r_{>}-r_{<} \leq r_{12} \leq r_{<}+r_{>}, 0 \leq j \leq 2 J$. Then for $\xi_{\ell}^{j}\left(r_{<}, r_{>}\right), A_{j}\left(r_{<}, r_{>}\right)$, and $R_{j, \ell}\left(r_{<}, r_{>}\right)$as given in (2.8), (2.9), and (2.11), the large $\ell$ behavior of $\psi_{\ell}$ (see (2.5)) is given by

$$
\psi_{\ell}\left(r_{<}, r_{>}\right)=\sum_{j=1}^{2 J-1} A_{j}\left(r_{<}, r_{>}\right) \xi_{\ell}^{j}\left(r_{<,} r_{>}\right)+R_{2 J-1, \ell}\left(r_{<}, r_{>}\right) .
$$

Furthermore, suppose that $A\left(r_{<}, r_{>}\right)$satisfies $A\left(\cdot, r_{>}\right) \in C^{N}\left(\mathbb{R}^{+}\right)$, and there exists $\tilde{A}: \mathbb{R}^{+} \rightarrow \mathbb{R}$ such that

$$
\tilde{A}\left(r_{>}\right) \geq\left|\left[\frac{\partial^{n}}{\partial r_{<}^{n}}\left|A\left(r_{<}, r_{>}\right)\right|^{2}\right]_{r_{<}=s}\right|, \forall s \in \mathbb{R}^{+}, n=0, \ldots, N .
$$

Then, for any $M<N$, and recalling the norms defined in (2.13),

$$
\begin{gathered}
\left\|A\left(r_{<}, r_{>}\right) \xi_{\ell}^{j}\left(r_{<}, r_{>}\right)\right\|_{a, b}^{2} \\
=\mathcal{O}\left(\ell^{-2 j-4}\right)\left[\sum_{m=0}^{M} \int_{0}^{\infty} r_{>}^{2 j+1+a+b} \frac{\left(r_{<}-r_{>}\right)^{m}}{m !}\left[\frac{\partial^{m}}{\partial r_{<}^{m}}\left|A\left(r_{<}, r_{>}\right)\right|^{2}\right]_{r_{<}=r_{>}} d r_{>}\right. \\
\left.\quad+\sum_{n=M+1}^{N} \mathcal{O}\left(\ell^{-n}\right) \int_{0}^{\infty} r_{>}^{2 j+1+a+b+n} \tilde{A}\left(r_{>}\right) d r_{>}\right] .
\end{gathered}
$$

Suppose that $I_{2 J}$, as given in (2.12), is finite. Then

$$
\lim _{\ell \rightarrow \infty} \ell^{2 J} R_{2 J-1, \ell}\left(r_{<}, r_{>}\right)=0 .
$$

If, in addition,

$$
\int_{0}^{\infty} \int_{0}^{r>} r_{<}^{4 J-1} I_{2 J}\left(r_{<}, r_{>}\right) r_{<}^{2} r_{>}^{2} d r_{<} d r_{>}<\infty
$$

then

$$
\lim _{\ell \rightarrow \infty} \ell^{4 J} \int_{0}^{\infty} \int_{0}^{r>}\left|R_{2 J-1, \ell}\left(r_{<}, r_{>}\right)\right|^{2} r_{<}^{2} r_{>}^{2} d r_{<} d r_{>}=0
$$

THEOREM 4.3. Let $\psi\left(r_{<}, r_{>}, r_{12}\right)$ be a function such that $\frac{\partial^{j}}{\partial r_{12}^{j}} \psi\left(r_{<}, r_{>}, r_{12}\right)$ exist for $r_{>}-r_{<} \leq r_{12} \leq r_{<}+r_{>}, 0 \leq j \leq 2 J+1$, and let $r_{\gamma} \in\left\{r_{<}, r_{>}\right\}$. Suppose 
$\frac{\partial}{\partial r_{\gamma}} \psi_{\ell}\left(r_{<}, r_{>}\right)$exist, then, with the same notation as in Theorem 4.2 , the large- $\ell$ behavior of $\frac{\partial}{\partial r_{\gamma}} \psi_{\ell}\left(r_{<}, r_{>}\right)$is given by

$$
\frac{\partial}{\partial r_{\gamma}} \psi_{\ell}\left(r_{<}, r_{>}\right)=\sum_{j=1}^{2 J} \frac{\partial}{\partial r_{\gamma}}\left(A_{j}\left(r_{<}, r_{>}\right) \xi_{\ell}^{j}\left(r_{<}, r_{>}\right)\right)+\frac{\partial}{\partial r_{\gamma}} R_{2 J, \ell}\left(r_{<}, r_{>}\right) .
$$

Furthermore, for $A\left(r_{<}, r_{>}\right)$and $\tilde{A}\left(r_{>}\right)$as in Theorem 4.2 , for any $M<N$,

$$
\begin{gathered}
\left\|A\left(r_{<}, r_{>}\right) \frac{\partial}{\partial r_{\gamma}} \xi_{\ell}^{j}\left(r_{<}, r_{>}\right)\right\|_{a, b}^{2} \\
=\mathcal{O}\left(\ell^{-2 j-2}\right)\left[\sum_{m=0}^{M} \int_{0}^{\infty} r_{>}^{2 j-1+a+b}\left(r_{<}-r_{>}\right)^{m}\left[\frac{\partial^{m}}{\partial r_{<}^{m}}\left|A\left(r_{<}, r_{>}\right)\right|^{2}\right]_{r_{<}=r_{>}} d r_{>}\right. \\
\left.+\sum_{n=M+1}^{N} \mathcal{O}\left(\ell^{-n}\right) \int_{0}^{\infty} r_{>}^{2 j+1+a+b} \tilde{A}\left(r_{>}\right) d r_{>}\right] .
\end{gathered}
$$

If $I_{2 J+1}<\infty$, then

$$
\lim _{\ell \rightarrow \infty} \ell^{2 J} \frac{\partial}{\partial r_{\gamma}} R_{2 J, \ell}\left(r_{<}, r_{>}\right)=0
$$

If, in addition,

$$
\int_{0}^{\infty} \int_{0}^{r>} r_{<}^{4 J-1} I_{2 J+1}\left(r_{<}, r_{>}\right) r_{<}^{2} r_{>}^{2} d r_{<} d r_{>}<\infty
$$

then

$$
\lim _{\ell \rightarrow \infty} \ell^{4 J} \int_{0}^{\infty} \int_{0}^{r_{>}}\left|\frac{\partial}{\partial r_{\gamma}} R_{2 J, \ell}\left(r_{<}, r_{>}\right)\right|^{2} r_{<}^{2} r_{>}^{2} d r_{<} d r_{>}=0
$$

These two results are analogous to Theorems 2 and 3 of [11], although formulated in a slightly different way.

\section{Proof of Theorem 3.1 using Theorems 4.2 and 4.3 .}

5.1. Preliminary lemmas. Before we prove the our main result using Theorems 4.2 and 4.3 , we require a few preliminary lemmas. The first of these is simply a Taylor expansion of a general, sufficiently regular, wavefunction.

LEMMA 5.1. Let $\psi\left(r_{<}, r_{>}, r_{12}\right)$ be a general wavefunction and suppose that $\frac{\partial^{j}}{\partial r_{12}^{j}} \psi\left(r_{<}, r_{>}, r_{12}\right)$ exist for $r_{>}-r_{<} \leq r_{12} \leq r_{<}+r_{>}, 0 \leq j \leq J$. Then $\psi\left(r_{<}, r_{>}, r_{12}\right)$ can be expanded as

$$
\begin{aligned}
\psi\left(r_{<}, r_{>}, r_{12}\right)= & \sum_{j=0}^{J-1} \frac{\left(r_{12}-\left(r_{>}-r_{<}\right)\right)^{j}}{j !}\left[\frac{\partial^{j}}{\partial r_{12}^{j}} \psi\left(r_{<}, r_{>}, r_{12}\right)\right]_{r_{12}=r_{>}-r_{<}} \\
& +\int_{r_{>}-r_{<}}^{r_{12}} \frac{\left(r_{12}-t\right)^{J-1}}{(J-1) !} \frac{\partial^{J}}{\partial r_{12}^{J}} \psi\left(r_{<}, r_{>}, t\right) d t .
\end{aligned}
$$

Copyright $@$ by SIAM. Unauthorized reproduction of this article is prohibited. 
Furthermore, for $\ell>0$,

$$
\psi_{\ell}\left(r_{<}, r_{>}\right)=\sum_{j=1}^{J-1} \xi_{\ell}^{j}\left(r_{<}, r_{>}\right) A_{j}\left(r_{<}, r_{>}\right)+R_{J-1, \ell}\left(r_{<}, r_{>}\right) .
$$

Proof. The first part is simply the application of Taylor's theorem about $\theta=0$ (which is equivalent to $r_{12}=r_{>}-r_{<}$) with the Cauchy form of the remainder.

The second part follows by projecting each term of the finite expansion onto $\Phi_{\ell}(\theta)$ to obtain the expression for $\psi_{\ell}\left(r_{<}, r_{>}\right)$in terms of (2.8), (2.9), and (2.11). In addition, we use that, for $\ell>0$, the term $\left[\frac{\partial^{j}}{\partial r_{12}^{j}} \psi\left(r_{<}, r_{>}, r_{12}\right)\right]_{r_{12}=r_{>}-r_{<}}$is simply a multiplicative factor as it is independent of $\theta$. For the same reason, for $\ell>0$, the term corresponding to $j=0$ contributes zero.

Note that it is this result which motivates the definitions (2.8)-(2.11).

Lemma 5.2. Let $\psi \in H^{1}$ be a general wavefunction. The following identities hold:

$$
\begin{aligned}
&\left\|\psi_{L}^{\perp}\right\|^{2}= 16 \pi^{2} \sum_{\ell=L+1}^{\infty} \int_{0}^{\infty} \int_{0}^{r_{>}}\left|\psi_{\ell}\left(r_{<}, r_{>}\right)\right|^{2} r_{<}^{2} r_{>}^{2} d r_{<} d r_{>}, \\
&\left\|\psi_{L}^{\perp}\right\|_{e}^{2}= 8 \pi^{2} \sum_{\ell=L+1}^{\infty} \int_{0}^{\infty} \int_{0}^{r>}\left(\left|\frac{\partial}{\partial r_{<}} \psi_{\ell}\left(r_{<}, r_{>}\right)\right|^{2}+\left|\frac{\partial}{\partial r_{>}} \psi_{\ell}\left(r_{<}, r_{>}\right)\right|^{2}\right) r_{<}^{2} r_{>}^{2} d r_{<} d r_{>} \\
&(5.3) \quad+8 \pi^{2} \sum_{\ell=L+1}^{\infty} \int_{0}^{\infty} \int_{0}^{r_{>}} \ell(\ell+1)\left(r_{<}^{2}+r_{>}^{2}\right)\left|\psi_{\ell}\left(r_{<}, r_{>}\right)\right|^{2} d r_{<} d r_{>} .
\end{aligned}
$$

Proof. Noting that $\int_{0}^{\pi}\left|\psi_{L}^{\frac{1}{L}}\right|^{2} \sin \theta d \theta=\sum_{\ell=L+1}^{\infty}\left|\psi_{\ell}\right|^{2}$, using spherical polar coordinates and integrating over the three angles that are independent of $\theta$ (essentially $\phi_{1}, \phi_{2}$, and $\theta_{1}$, leaving $\theta_{1}-\theta_{2}=: \theta$ ) gives a factor of $8 \pi^{2}$. We are hence left with only the radial integrals, and using the dominated convergence theorem gives

$$
\left\|\psi_{L}^{\perp}\right\|^{2}=8 \pi^{2} \sum_{\ell=L+1}^{\infty} \int_{0}^{\infty} \int_{0}^{\infty}\left|\psi_{\ell}\left(r_{1}, r_{2}\right)\right|^{2} r_{1}^{2} r_{2}^{2} d r_{1} d r_{2} .
$$

Using the general identity

$$
\int_{0}^{\infty} \int_{0}^{\infty} f\left(r_{1}, r_{2}\right) d r_{1} d r_{2}=\int_{0}^{\infty} \int_{0}^{r_{>}}\left(f\left(r_{<}, r_{>}\right)+f\left(r_{>}, r_{<}\right)\right) d r_{<} d r_{>}
$$

and the fact that $\left|\psi_{\ell}\left(r_{1}, r_{2}\right)\right|^{2} r_{1}^{2} r_{2}^{2}$ is symmetric in $r_{1}$ and $r_{2}$, gives the first result.

For the second case we use that, for symmetric functions $f\left(r_{<}, r_{>}, \theta\right), H_{0}:=$ $-\frac{1}{2}\left(\Delta_{1}+\Delta_{2}\right)$ may be rewritten [13] as $-\frac{1}{2}\left(\Delta_{1}^{\prime}+\Delta_{2}^{\prime}\right)$ where

$$
\Delta_{i}^{\prime}:=\frac{1}{r_{i}^{2}} \frac{\partial}{\partial r_{i}} r_{i}^{2} \frac{\partial}{\partial r_{i}}+\frac{1}{r_{i}^{2} \sin \theta} \frac{\partial}{\partial \theta} \sin \theta \frac{\partial}{\partial \theta}
$$

along with

$$
\frac{1}{\sin \theta} \frac{\partial}{\partial \theta} \sin \theta \frac{\partial}{\partial \theta} \Phi_{\ell}(\theta)=-\ell(\ell+1) \Phi_{\ell}(\theta)
$$

Copyright $@$ by SIAM. Unauthorized reproduction of this article is prohibited. 
to give

$$
H_{0} \psi_{L}^{\perp}=-\frac{1}{2} \sum_{\ell=L+1}^{\infty} \sum_{i=1}^{2}\left(\frac{1}{r_{i}^{2}} \frac{\partial}{\partial r_{i}} r_{i}^{2} \frac{\partial}{\partial r_{i}}-\frac{\ell(\ell+1)}{r_{i}^{2}}\right) \psi_{\ell}\left(r_{1}, r_{2}\right) \Phi_{\ell}(\theta),
$$

and, as with the previous case, we find

$$
\begin{aligned}
\left\|\psi_{L}^{\perp}\right\|_{e}^{2}= & -4 \pi^{2} \int_{0}^{\infty} \int_{0}^{\infty} \sum_{\ell=L+1}^{\infty} \sum_{i=1}^{2} \psi_{\ell}\left(r_{1}, r_{2}\right)\left(\frac{1}{r_{i}^{2}} \frac{\partial}{\partial r_{i}} r_{i}^{2} \frac{\partial}{\partial r_{i}}-\frac{\ell(\ell+1)}{r_{i}^{2}}\right) \psi_{\ell}\left(r_{1}, r_{2}\right)^{*} \\
& \times r_{1}^{2} r_{2}^{2} d r_{1} d r_{2} .
\end{aligned}
$$

Again, using the dominated convergence theorem, and integrating the first term by parts, gives

$$
\left\|\psi_{L}^{\perp}\right\|_{e}^{2}=4 \pi^{2} \sum_{\ell=L+1}^{\infty} \int_{0}^{\infty} \int_{0}^{\infty} \sum_{i=1}^{2}\left(\left|\frac{\partial}{\partial r_{i}} \psi_{\ell}\left(r_{1}, r_{2}\right)\right|^{2}+\frac{\ell(\ell+1)}{r_{i}^{2}}\left|\psi_{\ell}\left(r_{1}, r_{2}\right)\right|^{2}\right) r_{1}^{2} r_{2}^{2} d r_{1} d r_{2} .
$$

Noting that the integrand is once again symmetric in $r_{1}$ and $r_{2}$ and using (5.4) gives the result.

LEMMA 5.3. Let $\psi$ be a general wavefunction and suppose that $\frac{\partial^{j}}{\partial r_{12}^{j}} \psi\left(r_{<}, r_{>}, r_{12}\right)$ exist for $r_{>}-r_{<} \leq r_{12} \leq r_{<}+r_{>}, 0 \leq j \leq J$, and that $\frac{\partial}{\partial r_{\gamma}} \psi_{\ell}\left(r_{<}, r_{>}\right)$exist for $r_{\gamma} \in\left\{r_{<}, r_{>}\right\}$. Then, using the notation of (2.8)-(2.11) the norms (2.13) of the partial waves (2.5) and their derivatives satisfy

$$
\begin{aligned}
& \left\|\psi_{\ell}\left(r_{<}, r_{>}\right)\right\|_{a, b}^{2} \leq \sum_{i, j=1}^{J-1}\left\|A_{i}\left(r_{<}, r_{>}\right) \xi_{\ell}^{i}\left(r_{<}, r_{>}\right)\right\|_{a, b}\left\|A_{j}\left(r_{<}, r_{>}\right) \xi_{\ell}^{j}\left(r_{<}, r_{>}\right)\right\|_{a, b} \\
& \quad+2 \sum_{k=1}^{J-1}\left\|A_{k}\left(r_{<}, r_{>}\right) \xi_{\ell}^{k}\left(r_{<}, r_{>}\right)\right\|_{a, b}\left\|R_{J-1, \ell}\left(r_{<}, r_{>}\right)\right\|_{a, b}+\left\|R_{J-1, \ell}\left(r_{<}, r_{>}\right)\right\|_{a, b}^{2}
\end{aligned}
$$

$$
\begin{aligned}
& \text { and }\left\|\frac{\partial}{\partial r_{\gamma}} \psi_{\ell}\left(r_{<}, r_{>}\right)\right\|_{a, b}^{2} \\
& \leq \sum_{i, j=1}^{J-1}\left[\left\|\xi_{\ell}^{i}\left(r_{<}, r_{>}\right) \frac{\partial}{\partial r_{\gamma}} A_{i}\left(r_{<}, r_{>}\right)\right\|_{a, b}\left\|\xi_{\ell}^{j}\left(r_{<}, r_{>}\right) \frac{\partial}{\partial r_{\gamma}} A_{j}\left(r_{<}, r_{>}\right)\right\|_{a, b}\right. \\
& +2\left\|\xi_{\ell}^{i}\left(r_{<}, r_{>}\right) \frac{\partial}{\partial r_{\gamma}} A_{i}\left(r_{<}, r_{>}\right)\right\|_{a, b}\left\|A_{j}\left(r_{<}, r_{>}\right) \frac{\partial}{\partial r_{\gamma}} \xi_{\ell}^{j}\left(r_{<}, r_{>}\right)\right\|_{a, b} \\
& \left.+\left\|A_{i}\left(r_{<}, r_{>}\right) \frac{\partial}{\partial r_{\gamma}} \xi_{\ell}^{i}\left(r_{<}, r_{>}\right)\right\|\left\|_{a, b}\right\| A_{j}\left(r_{<}, r_{>}\right) \frac{\partial}{\partial r_{\gamma}} \xi_{\ell}^{j}\left(r_{<}, r_{>}\right) \|_{a, b}\right] \\
& +2\left\|\frac{\partial}{\partial r_{\gamma}} R_{J-1, \ell}\left(r_{<}, r_{>}\right)\right\| \sum_{a, b}^{J-1}\left\|A_{k}\left(r_{<}, r_{>}\right) \frac{\partial}{\partial r_{\gamma}} \xi_{\ell}^{k}\left(r_{<}, r_{>}\right)\right\|_{a, b} \\
& +2\left\|\frac{\partial}{\partial r_{\gamma}} R_{J-1, \ell}\left(r_{<}, r_{>}\right)\right\|_{a, b} \sum_{k=1}^{J-1}\left\|\xi_{\ell}^{k}\left(r_{<}, r_{>}\right) \frac{\partial}{\partial r_{\gamma}} A_{k}\left(r_{<}, r_{>}\right)\right\|_{a, b} \\
& +\left\|\frac{\partial}{\partial r_{\gamma}} R_{J-1, \ell}\left(r_{<}, r_{>}\right)\right\|_{a, b}^{2} .
\end{aligned}
$$

Copyright $@$ ㅇ by SIAM. Unauthorized reproduction of this article is prohibited. 
Proof. It is immediately clear from (5.1) that, for $\ell>0$, we have

$$
\begin{aligned}
\left|\psi_{\ell}\left(r_{<}, r_{>}\right)\right|^{2}= & \sum_{i, j=1}^{J-1} A_{i}\left(r_{<}, r_{>}\right) \xi_{\ell}^{i}\left(r_{<}, r_{>}\right) A_{j}\left(r_{<}, r_{>}\right)^{*} \xi_{\ell}^{j}\left(r_{<}, r_{>}\right)^{*} \\
& +2 \operatorname{Re}\left(R_{J-1, \ell}\left(r_{<}, r_{>}\right)^{*} \sum_{k=1}^{J-1} A_{k}\left(r_{<}, r_{>}\right) \xi_{\ell}^{k}\left(r_{<}, r_{>}\right)\right) \\
& +\left|R_{J-1, \ell}\left(r_{<,} r_{>}\right)\right|^{2}, \quad \text { and }
\end{aligned}
$$

$$
\begin{aligned}
\left|\frac{\partial}{\partial r_{\gamma}} \psi_{\ell}\left(r_{<}, r_{>}\right)\right|^{2}= & \sum_{i, j=1}^{J-1} \frac{\partial}{\partial r_{\gamma}}\left(A_{i}\left(r_{<,} r_{>}\right) \xi_{\ell}^{i}\left(r_{<}, r_{>}\right)\right) \frac{\partial}{\partial r_{\gamma}}\left(A_{j}\left(r_{<}, r_{>}\right) \xi_{\ell}^{j}\left(r_{<}, r_{>}\right)\right)^{*} \\
& +2 \operatorname{Re}\left(\frac{\partial}{\partial r_{\gamma}} R_{J-1, \ell}\left(r_{<}, r_{>}\right)^{*} \sum_{k=1}^{2 J-1} \frac{\partial}{\partial r_{\gamma}}\left(A_{k}\left(r_{<}, r_{>}\right) \xi_{\ell}^{k}\left(r_{<}, r_{>}\right)\right)\right) \\
& +\left|\frac{\partial}{\partial r_{\gamma}} R_{J-1, \ell}\left(r_{<,} r_{>}\right)\right|^{2} .
\end{aligned}
$$

The results then follow by inserting these expressions into the norms (2.13), using the linearity of the integrals, and applying Cauchy-Schwarz. In addition, the second expression uses the product rule for differentiation.

5.2. Proof of Theorem 3.1. We prove our main result using the lemmas from the previous section, along with Theorems 4.2 and 4.3 .

Proof. We begin with the case of $\left\|\psi_{L}^{\perp}\right\|^{2}$ and by (5.2) we see that $\left\|\psi_{L}^{\perp}\right\|^{2}=$ $16 \pi^{2} \sum_{\ell=L+1}^{\infty}\left\|\psi_{\ell}\left(r_{<}, r_{>}\right)\right\|_{2,2}^{2}$. Lemma 5.3 with $J=4$ gives

$$
\begin{aligned}
\left\|\psi_{\ell}\left(r_{<}, r_{>}\right)\right\|_{2,2}^{2} \leq & \sum_{i, j=1}^{3}\left\|A_{i}\left(r_{<}, r_{>}\right) \xi_{\ell}^{i}\left(r_{<}, r_{>}\right)\right\|_{2,2}\left\|A_{j}\left(r_{<}, r_{>}\right) \xi_{\ell}^{j}\left(r_{<}, r_{>}\right)\right\|_{2,2} \\
& +2 \sum_{k=1}^{3}\left\|A_{k}\left(r_{<}, r_{>}\right) \xi_{\ell}^{k}\left(r_{<}, r_{>}\right)\right\|_{2,2}\left\|R_{3, \ell}\left(r_{<}, r_{>}\right)\right\|_{2,2} \\
& +\left\|R_{3, \ell}\left(r_{<}, r_{>}\right)\right\|_{2,2}^{2} .
\end{aligned}
$$

By Theorem 4.2 with $J=2$, and in particular by (4.4), we have that

$$
\lim _{\ell \rightarrow \infty} \ell^{8}\left\|R_{3, \ell}\left(r_{<}, r_{>}\right)\right\|_{2,2}^{2}=\lim _{\ell \rightarrow \infty} \ell^{8} \int_{0}^{\infty} \int_{0}^{r>}\left|R_{3, \ell}\left(r_{<}, r_{>}\right)\right|^{2} r_{<}^{2} r_{>}^{2} d r_{<} d r_{>}=0
$$

and hence $\lim _{\ell \rightarrow \infty} \ell^{4}\left\|R_{3, \ell}\left(r_{<}, r_{>}\right)\right\|_{2,2}=0$; i.e., $\left\|R_{3, \ell}\left(r_{<}, r_{>}\right)\right\|_{2,2}=o\left(l^{-4}\right)$.

The same theorem shows (choosing $A\left(r_{<}, r_{>}\right)=A_{j}\left(r_{<}, r_{>}\right)$and $a=b=2$ in (4.2)) that $\left\|A_{j}\left(r_{<}, r_{>}\right) \xi_{\ell}^{j}\left(r_{<}, r_{>}\right)\right\|_{2,2}=\mathcal{O}\left(\ell^{-j-2}\right)$, and it is therefore clear that the slowest decaying term comes from the $i=j=1$ term in the first sum of (5.11), with all other terms decaying at least one order of $\ell$ faster. Theorem 4.2 also shows that (choosing $A\left(r_{<}, r_{>}\right)=A_{1}\left(r_{<}, r_{>}\right), M=0, a=b=2$ and $j=1$ in (4.2))

$$
\left\|A_{1}\left(r_{<}, r_{>}\right) \xi_{\ell}^{1}\left(r_{<}, r_{>}\right)\right\|_{2,2}^{2}=\mathcal{O}\left(\ell^{-6}\right) \int_{0}^{\infty} r_{>}^{7}\left[\left|A_{1}\left(r_{<}, r_{>}\right)\right|^{2}\right]_{r_{<}=r_{>}} d r_{>}+\mathcal{O}\left(\ell^{-7}\right)
$$

Copyright $@$ by SIAM. Unauthorized reproduction of this article is prohibited. 
By the definition of $A_{j}\left(r_{<}, r_{>}\right)$in (2.9), we have that

$$
\begin{aligned}
{\left[\left|A_{1}\left(r_{<}, r_{>}\right)\right|^{2}\right]_{r_{<}=r_{>}} } & =\left[\left[\frac{\partial}{\partial r_{12}} \psi\left(r_{<}, r_{>}, r_{12}\right)\right]_{r_{12}=r_{>}-r_{<}}\right]_{r_{<}=r_{>}} \\
& =\left[\frac{\partial}{\partial r_{12}} \psi\left(r_{>}, r_{>}, r_{12}\right)\right]_{r_{12}=0} .
\end{aligned}
$$

Using the Kato cusp condition without spherical averaging [12] (see also Appendix E of [11]),

$$
\left[\frac{\partial}{\partial r_{12}} \psi\left(r_{>}, r_{>}, r_{12}\right)\right]_{r_{12}=0}=\frac{1}{2} \psi\left(r_{>}, r_{>}, 0\right)
$$

gives (3.1) up to a constant, which will be computed later.

The proof of (3.2) is analogous, noting that (5.3) shows that

$$
\begin{aligned}
\left\|\psi_{L}^{\perp}\right\|_{e}^{2}=8 \pi^{2} \sum_{\ell=L+1}^{\infty}( & \left\|\frac{\partial}{\partial r_{<}} \psi_{\ell}\left(r_{<}, r_{>}\right)\right\|_{2,2}+\left\|\frac{\partial}{\partial r_{>}} \psi_{\ell}\left(r_{<}, r_{>}\right)\right\|_{2,2} \\
& \left.+\ell(\ell+1)\left[\left\|\psi_{\ell}\left(r_{<}, r_{>}\right)\right\|_{2,0}+\left\|\psi_{\ell}\left(r_{<}, r_{>}\right)\right\|_{0,2}\right]\right) .
\end{aligned}
$$

For the terms involving $\psi_{\ell}\left(r_{<}, r_{>}\right)$we use Theorem 4.2 with $J=2$ and in particular (4.2) with $M=0, A\left(r_{<}, r_{>}\right)=A_{1}\left(r_{<}, r_{>}\right), j=1$ and either $a=2, b=0$ or $a=0, b=$ 2 , giving

$$
\left\|A_{1}\left(r_{<}, r_{>}\right) \xi_{\ell}^{1}\left(r_{<}, r_{>}\right)\right\|_{2,0}^{2}=\mathcal{O}\left(\ell^{-6}\right) \int_{0}^{\infty} r_{>}^{5}\left[\left|A_{1}\left(r_{<}, r_{>}\right)\right|^{2}\right]_{r_{<}=r_{>}} d r_{>}+\mathcal{O}\left(\ell^{-7}\right)
$$

$$
\left\|A_{1}\left(r_{<}, r_{>}\right) \xi_{\ell}^{1}\left(r_{<}, r_{>}\right)\right\|_{0,2}^{2}=\mathcal{O}\left(\ell^{-6}\right) \int_{0}^{\infty} r_{>}^{5}\left[\left|A_{1}\left(r_{<}, r_{>}\right)\right|^{2}\right]_{r_{<}=r_{>}} d r_{>}+\mathcal{O}\left(\ell^{-7}\right) .
$$

For the other terms in (5.13), we use Lemma 5.3 with $J=3$ to obtain

$$
\begin{aligned}
& \left\|\frac{\partial}{\partial r_{\gamma}} \psi_{\ell}\left(r_{<}, r_{>}\right)\right\|_{2,2}^{2} \\
& \leq \sum_{i, j=1}^{2}\left[\left\|\xi_{\ell}^{i}\left(r_{<}, r_{>}\right) \frac{\partial}{\partial r_{\gamma}} A_{i}\left(r_{<}, r_{>}\right)\right\|_{2,2}\left\|\xi_{\ell}^{j}\left(r_{<}, r_{>}\right) \frac{\partial}{\partial r_{\gamma}} A_{j}\left(r_{<}, r_{>}\right)\right\|_{2,2}\right. \\
& \quad+2\left\|\xi_{\ell}^{i}\left(r_{<}, r_{>}\right) \frac{\partial}{\partial r_{\gamma}} A_{i}\left(r_{<}, r_{>}\right)\right\|_{2,2}\left\|A_{j}\left(r_{<}, r_{>}\right) \frac{\partial}{\partial r_{\gamma}} \xi_{\ell}^{j}\left(r_{<}, r_{>}\right)\right\|_{2,2} \\
& \left.\quad+\left\|A_{i}\left(r_{<}, r_{>}\right) \frac{\partial}{\partial r_{\gamma}} \xi_{\ell}^{i}\left(r_{<}, r_{>}\right)\right\|_{2,2}\left\|A_{j}\left(r_{<}, r_{>}\right) \frac{\partial}{\partial r_{\gamma}} \xi_{\ell}^{j}\left(r_{<}, r_{>}\right)\right\|_{2,2}\right] \\
& +2\left\|\frac{\partial}{\partial r_{\gamma}} R_{J-1, \ell}\left(r_{<}, r_{>}\right)\right\|_{2,2} \sum_{k=1}^{2}\left\|A_{k}\left(r_{<}, r_{>}\right) \frac{\partial}{\partial r_{\gamma}} \xi_{\ell}^{k}\left(r_{<}, r_{>}\right)\right\|_{2,2} \\
& +2\left\|\frac{\partial}{\partial r_{\gamma}} R_{J-1, \ell}\left(r_{<}, r_{>}\right)\right\|_{2,2} \sum_{k=1}^{2 J-1}\left\|\xi_{\ell}^{k}\left(r_{<}, r_{>}\right) \frac{\partial}{\partial r_{\gamma}} A_{k}\left(r_{<}, r_{>}\right)\right\|_{2,2} \\
& +\left\|\frac{\partial}{\partial r_{\gamma}} R_{2, \ell}\left(r_{<}, r_{>}\right)\right\|_{2,2}^{2} \cdot
\end{aligned}
$$

Copyright (c) by SIAM. Unauthorized reproduction of this article is prohibited. 
By Theorem 4.3 with $J=1$ (and in particular (4.8)) we find $\left\|\frac{\partial}{\partial r_{\gamma}} R_{2, \ell}\left(r_{<}, r_{>}\right)\right\|_{2,2}^{2}=$ $o\left(\ell^{-4}\right)$. Furthermore, Theorem 4.2 shows that, for $J=2, A\left(r_{<}, r_{>}\right)=\frac{\partial}{\partial r_{\gamma}} A_{j}\left(r_{<}, r_{>}\right)$, $a=b=2$, and $j=1,2$ in (4.2),

$$
\begin{aligned}
& \left\|\xi_{\ell}^{1}\left(r_{<}, r_{>}\right) \frac{\partial}{\partial r_{\gamma}} A_{1}\left(r_{<}, r_{>}\right)\right\|_{2,2}^{2}=\mathcal{O}\left(\ell^{-6}\right), \text { and } \\
& \left\|\xi_{\ell}^{2}\left(r_{<}, r_{>}\right) \frac{\partial}{\partial r_{\gamma}} A_{2}\left(r_{<}, r_{>}\right)\right\|_{2,2}^{2}=\mathcal{O}\left(\ell^{-8}\right) .
\end{aligned}
$$

Using (4.6) of Theorem 4.3 with $J=1, A\left(r_{<}, r_{>}\right)=A_{2}\left(r_{<}, r_{>}\right), j=2$, and $a=b=2$ gives

$$
\left\|A_{2}\left(r_{<}, r_{>}\right) \frac{\partial}{\partial r_{\gamma}} \xi_{\ell}^{2}\left(r_{<}, r_{>}\right)\right\|_{2,2}^{2}=\mathcal{O}\left(\ell^{-6}\right)
$$

Hence it is only necessary to consider the term $\left\|A_{1}\left(r_{<}, r_{>}\right) \frac{\partial}{\partial r_{\gamma}} \xi_{\ell}^{1}\left(r_{<}, r_{>}\right)\right\|_{2,2}$ in (5.16). Once again using (4.6) with $J=1, A\left(r_{<}, r_{>}\right)=A_{1}\left(r_{<}, r_{>}\right), j=1, a=b=2$, and $M=0$ gives

$$
\left\|A_{1}\left(r_{<}, r_{>}\right) \frac{\partial}{\partial r_{\gamma}} \xi_{\ell}^{1}\left(r_{<}, r_{>}\right)\right\|_{2,2}^{2}=\mathcal{O}\left(\ell^{-6}\right) \int_{0}^{\infty} r_{>}^{5}\left[\left|A_{1}\left(r_{<}, r_{>}\right)\right|^{2}\right]_{r_{<}=r_{>}} d r_{>}+\mathcal{O}\left(\ell^{-7}\right)
$$

As before, using the definition of $A_{j}\left(r_{<}, r_{>}\right)$and the cusp condition gives (3.2) up to a constant.

Calculation of constants. We begin by noting that, for $\ell>0, \xi_{\ell}^{1}\left(r_{<}, r_{>}\right)=$ $\int_{0}^{\pi}\left(r_{12}-\left(r_{>}-r_{<}\right)\right) \Phi_{\ell}(\theta) \sin \theta d \theta$ is given by

$$
\xi_{\ell}^{1}\left(r_{<}, r_{>}\right)=\left(\ell+\frac{1}{2}\right)^{-1 / 2} f_{\ell}^{1}\left(r_{<}, r_{>}\right)=\left(\ell+\frac{1}{2}\right)^{-1 / 2} r_{<}^{\ell} r_{<}^{-\ell-1}\left(\frac{r_{<}^{2}}{2 \ell+3}-\frac{r_{>}^{2}}{2 \ell-1}\right) .
$$

Hence

$$
\begin{aligned}
& \frac{\partial}{\partial r_{<}} \xi_{\ell}^{1}\left(r_{<}, r_{>}\right)=\left(\ell+\frac{1}{2}\right)^{-1 / 2} r_{>}^{-\ell-1}\left(\frac{r_{<}^{\ell+1}(\ell+2)}{2 \ell+3}-\frac{r_{>}^{2} r_{<}^{\ell-1} \ell}{2 \ell-1}\right), \quad \text { and } \\
& \frac{\partial}{\partial r_{>}} \xi_{\ell}^{1}\left(r_{<}, r_{>}\right)=\left(\ell+\frac{1}{2}\right)^{-1 / 2} r_{<}^{\ell}\left(\frac{r_{<}^{2} r_{>}^{-\ell-2}(-\ell-1)}{2 \ell+3}-\frac{r_{>}^{-\ell}(-\ell+1)}{2 \ell-1}\right) .
\end{aligned}
$$

It follows that

$$
\begin{aligned}
\int_{0}^{r>} r_{<}^{a} \mid & \left.\xi_{\ell}^{1}\left(r_{<}, r_{>}\right)\right|^{2} d r_{<} \\
& =r_{>}^{2 \ell+5+a} \frac{16\left(20 \ell^{2}+(44+12 a) \ell+2 a^{2}+14 a+21\right)}{(2 \ell+3)^{2}(2 \ell-1)^{2}(2 \ell+5+a)(2 \ell+3+a)(2 \ell+1+a)(2 \ell+1)}
\end{aligned}
$$

Copyright (c) by SIAM. Unauthorized reproduction of this article is prohibited. 
and so, setting $B_{n}:=\int_{0}^{\infty} r_{>}^{n}\left|A_{1}\left(r_{<}, r_{>}\right)\right|^{2} d r_{>}$,

$$
\begin{aligned}
& \left\|A_{1}\left(r_{<}, r_{>}\right) \xi_{\ell}^{1}\left(r_{<}, r_{>}\right)\right\|_{2,2}^{2}=\frac{16(10 \ell+19)}{(2 \ell+3)^{2}(2 \ell-1)^{2}(2 \ell+7)(2 \ell+5)(2 \ell+1)} B_{7} \\
& \left\|A_{1}\left(r_{<}, r_{>}\right) \xi_{\ell}^{1}\left(r_{<}, r_{>}\right)\right\|_{2,0}^{2}=\frac{16(10 \ell+19)}{(2 \ell+3)^{2}(2 \ell-1)^{2}(2 \ell+7)(2 \ell+5)(2 \ell+1)} B_{5} \\
& \left\|A_{1}\left(r_{<}, r_{>}\right) \xi_{\ell}^{1}\left(r_{<}, r_{>}\right)\right\|_{0,2}^{2}=\frac{16(10 \ell+7)}{(2 \ell+3)^{2}(2 \ell-1)^{2}(2 \ell+5)(2 \ell+1)^{2}} B_{5} .
\end{aligned}
$$

In a similar fashion, we find that

$$
\begin{aligned}
& \left\|A_{1}\left(r_{<}, r_{>}\right) \frac{\partial}{\partial r_{<}} \xi_{\ell}^{1}\left(r_{<}, r_{>}\right)\right\|_{2,2}^{2}=\frac{8\left(4 \ell^{3}+10 \ell^{2}+4 \ell+1\right)}{(2 \ell+3)^{2}(2 \ell-1)^{2}(2 \ell+5)(2 \ell+1)^{2}} B_{5} \\
& \left\|A_{1}\left(r_{<}, r_{>}\right) \frac{\partial}{\partial r_{>}} \xi_{\ell}^{1}\left(r_{<}, r_{>}\right)\right\|_{2,2}^{2}=\frac{8\left(4 \ell^{3}-6 \ell^{2}-8 \ell+17\right)}{(2 \ell+3)^{2}(2 \ell-1)^{2}(2 \ell+5)(2 \ell+1)^{2}} B_{5} .
\end{aligned}
$$

The leading term in $\left\|\psi_{L}^{\perp}\right\|^{2}$ is $16 \pi^{2}\left\|A_{1}\left(r_{<}, r_{>}\right) \xi_{\ell}^{1}\left(r_{<}, r_{>}\right)\right\|_{2,2}^{2}$, and hence the coefficient of the leading order term is

$$
\ell^{-6} 16 \pi^{2} \frac{160}{2^{7}} \int_{0}^{\infty} r_{>}^{7}\left|A_{1}\left(r_{<}, r_{>}\right)\right|^{2} d r_{>}=5 \pi^{2} \ell^{-6} \int_{0}^{\infty} r^{7}|\psi(r, r, 0)|^{2} d r
$$

where we have used the cusp condition (5.12). Similarly, the leading term in $\left\|\psi_{L}^{\perp}\right\|_{e}^{2}$ is

$$
\begin{aligned}
& 8 \pi^{2}\left(\left\|A_{1}\left(r_{<}, r_{>}\right) \xi_{\ell}^{1}\left(r_{<}, r_{>}\right)\right\|_{2,2}^{2}+\left\|A_{1}\left(r_{<}, r_{>}\right) \frac{\partial}{\partial r_{>}} \xi_{\ell}^{1}\left(r_{<}, r_{>}\right)\right\|_{2,2}^{2}\right. \\
& \left.\quad+\ell(\ell+1)\left[\left\|A_{1}\left(r_{<}, r_{>}\right) \xi_{\ell}^{1}\left(r_{<}, r_{>}\right)\right\|_{2,0}^{2}+\left\|A_{1}\left(r_{<}, r_{>}\right) \xi_{\ell}^{1}\left(r_{<}, r_{>}\right)\right\|_{0,2}^{2}\right]\right)
\end{aligned}
$$

which, combining the above, is given by

$$
8 \pi^{2} \frac{192\left(2 \ell^{3}+7 \ell^{2}+6 \ell+1\right)}{(2 \ell+3)^{2}(2 \ell-1)^{2}(2 \ell+7)(2 \ell+5)(2 \ell+1)} \int_{0}^{\infty} r_{>}^{5}\left|A_{1}\left(r_{<}, r_{>}\right)\right|^{2} d r_{>},
$$

and the leading order term is thus

$$
8 \pi^{2} \ell^{-4} \frac{192 \cdot 2}{2^{7}} \int_{0}^{\infty} r_{>}^{5}\left|A_{1}\left(r_{<,}, r_{>}\right)\right|^{2} d r_{>}=6 \pi^{2} \ell^{-4} \int_{0}^{\infty} r^{5}|\psi(r, r, 0)|^{2} d r
$$

where we have once again used the cusp condition (5.12). We have therefore shown (3.1) and (3.2) and it remains to show (3.3). For this we note that

$$
\frac{1}{n}(L+1)^{-n+1}=\int_{L+1}^{\infty} \ell^{-n} \leq \sum_{\ell=L+1}^{\infty} \ell^{-n} \leq \int_{L+1}^{\infty}(\ell-1)^{-n}=\frac{1}{n} L^{-n+1}
$$

and applying this to the leading term of $E_{L}$, which by Lemma 4.1 and the above decay rate estimates is given by the leading term of $\left\|\psi_{L}^{\perp}\right\|_{e}^{2}$, gives the result.

Note that Theorem 3.1 gives the same leading order result as [11].

Copyright (c) by SIAM. Unauthorized reproduction of this article is prohibited. 
6. Proof of Theorems 4.2 and 4.3. It remains to prove Theorems 4.2 and 4.3 . We begin by noting that (4.1) and (4.5) follow directly from (5.1) and term-by-term differentiation.

The remainder of the proof proceeds as follows: First we expand $\xi_{\ell}^{j}\left(r_{<}, r_{>}\right)$and its derivatives in $f_{\ell}^{k}\left(r_{<}, r_{>}\right)$, projections of $r_{12}^{2 k-1}$, for which we derive an explicit form (Lemma 6.1). This form allows us to determine a bound on the decay rates of the norms in (4.2) and (4.6) in terms of norms involving $f_{\ell}^{k}\left(r_{<}, r_{>}\right)$and its derivatives (see $(6.21)-(6.23))$. We then use the fact that the $f_{\ell}^{k}\left(r_{<}, r_{>}\right)$are strongly peaked on the line $r_{<}=r_{>}$(Lemma 6.5) and an expansion of the remaining terms in the integrals (Lemma 6.6) to make the $r_{<}$integral explicit. Finally, we use these explicit forms (Lemma 6.7) to derive the required decay rates (Corollary 6.10).

The rest of the section is devoted to proof of a bound on the remainder terms $R_{J, \ell}\left(r_{<}, r_{>}\right)$. Firstly we state the result under assumptions on the boundedness of $\theta$ derivatives of $R_{J}\left(r_{<}, r_{>}, r_{12}\right)$ (Lemmas 6.12 and 6.13). We then prove the validity of these assumptions by solving a recursion relation (see Lemma 6.14) explicitly enough to obtain the required bounds.

6.1. Expansion of $\xi_{\ell}^{j}\left(r_{<}, r_{>}\right)$. For this section we follow the proof of Hill's Theorem 2 (specifically [11, p. 1180]) by noting that

$$
\xi_{\ell}^{j}\left(r_{<}, r_{>}\right)=\frac{1}{j !} \sum_{k=0}^{j} \int_{0}^{\pi}\left(\begin{array}{l}
j \\
k
\end{array}\right)(-1)^{k}\left(r_{>}-r_{<}\right)^{j-k} r_{12}^{k} \Phi_{\ell}(\theta) \sin \theta d \theta .
$$

Since the Legendre polynomial expansion of $r_{12}^{2 k}$ terminates at $\ell=k$, taking $\ell>j / 2$ gives

$\xi_{\ell}^{j}\left(r_{<}, r_{>}\right)=\frac{1}{j !} \sum_{k=0}^{\lfloor(j+1) / 2\rfloor}\left(\begin{array}{c}j \\ 2 k-1\end{array}\right)(-1)^{j-2 k+1}\left(r_{>}-r_{<}\right)^{j-2 k+1} \int_{0}^{\pi} r_{12}^{2 k-1} \Phi_{\ell}(\theta) \sin \theta d \theta$,

where the notation $\lfloor n\rfloor$ means the greatest integer $m \leq n$. We would now like to show that all terms in this sum decay at the same rate, i.e., that the rate is independent of $k$.

The first stage of this is to write the Legendre polynomial expansions of odd powers of $r_{12}$ as

$$
r_{12}^{2 k-1}=: \sum_{\ell=0}^{\infty}\left(\ell+\frac{1}{2}\right)^{-1 / 2} f_{\ell}^{k}\left(r_{<}, r_{>}\right) \Phi_{\ell}(\theta),
$$

from which it immediately follows (using the orthonormality of $\Phi_{\ell}(\theta)$ ) that

$$
\int_{0}^{\pi} r_{12}^{2 k-1} \Phi_{\ell}(\theta) \sin \theta d \theta=\left(\ell+\frac{1}{2}\right)^{-1 / 2} f_{\ell}^{k}\left(r_{<}, r_{>}\right) .
$$

We would therefore like to find an explicit form for the $f_{\ell}^{k}\left(r_{<}, r_{>}\right)$, which will allow us to estimate the necessary decay rates.

6.2. An explicit form for $f_{\ell}^{k}\left(r_{<}, r_{>}\right)$. We begin by considering the form of $f_{\ell}^{k}\left(r_{<}, r_{>}\right)$, the (up to a multiplicative constant) projection of $r_{12}^{2 k-1}$ onto the $\ell$ th angular momentum eigenstate $\Phi_{\ell}(\theta)$. Note that this step is not performed in [11], where an estimate on the decay is used instead. For our purposes an explicit form is much more useful, especially regarding the derivatives of $f_{\ell}^{k}\left(r_{<}, r_{>}\right)$. 
LEMMA 6.1. With the convention that an empty product takes the value 1 ,

$$
f_{\ell}^{k}\left(r_{<}, r_{>}\right)=r_{<}^{\ell} r_{>}^{-\ell-1} \sum_{j=0}^{k} \frac{a_{j}^{k} r_{<}^{2(k-j)} r_{>}^{2 j}}{\prod_{m=1}^{k-j}(2 \ell+2 m+1) \prod_{n=1}^{j}(2 \ell-2 n+1)},
$$

where $a_{j}^{k} \in \mathbb{Z}$ are given by

$$
a_{0}^{k}=\prod_{i=1}^{k}(2 k-2 i+1), \quad a_{j}^{k}=(-1)^{j}\left(\begin{array}{c}
k \\
j
\end{array}\right) a_{0}^{k}, j=1, \ldots, k .
$$

We prove Lemma 6.1 in a number of stages. Firstly we show that LEMMA 6.2.

$$
f_{\ell}^{k}\left(r_{<}, r_{>}\right)=r_{<}^{\ell} r_{>}^{-\ell-1} \sum_{j=0}^{k} c_{j}^{k, \ell} r_{<}^{2(k-j)} r_{>}^{2 j},
$$

where $c_{j}^{k, \ell}$ is given by the recursion relation

$$
c_{j}^{k+1, \ell}=c_{j}^{k, \ell}+c_{j-1}^{k, \ell}-\frac{2 \ell}{2 \ell-1} c_{j-1}^{k, \ell-1}-\frac{2(\ell+1)}{2 \ell+3} c_{j}^{k, \ell+1}
$$

and the initial condition $c_{0}^{0, \ell}=1$.

We then derive a suitable expression for $c_{j}^{k, \ell}$ which proves Lemma 6.1.

Proof. Note that, by (2.2) and (6.2),

$$
r_{12}^{2 k+1}=\left(r_{<}^{2}+r_{>}^{2}-2 r_{<} r_{>} \cos \theta\right) \sum_{\ell=0}^{\infty}\left(\ell+\frac{1}{2}\right)^{-1 / 2} f_{\ell}^{k}\left(r_{<}, r_{>}\right) \Phi_{\ell}(\theta) .
$$

In order to determine the action of $\cos \theta$ on $\Phi_{\ell}(\theta)$, we recall the definition (2.4) of $\Phi_{\ell}(\theta)$ and the recursion relation for the Legendre polynomials [1]

$$
(\ell+1) P_{\ell+1}(\cos \theta)=(2 \ell+1) \cos \theta P_{\ell}(\cos \theta)-\ell P_{\ell-1}(\cos \theta) .
$$

It follows from (6.7) that

$$
\cos \theta\left(\ell+\frac{1}{2}\right)^{1 / 2} P_{\ell}=\frac{\ell+1}{2 \ell+1}\left(\ell+\frac{1}{2}\right)^{1 / 2} P_{\ell+1}+\frac{\ell}{2 \ell+1}\left(\ell+\frac{1}{2}\right)^{1 / 2} P_{\ell-1}
$$

and hence by $(2.4)$

$$
\cos \theta \Phi_{\ell}(\theta)=\frac{\ell+1}{[(2 \ell+1)(2 \ell+3)]^{1 / 2}} \Phi_{\ell+1}(\theta)+\frac{\ell}{[(2 \ell+1)(2 \ell-1)]^{1 / 2}} \Phi_{\ell-1}(\theta) .
$$

Inserting (6.8) into (6.6) and comparing coefficients of $\Phi_{\ell}(\theta)$ with (6.2) gives the recursion relation

$$
\begin{aligned}
f_{\ell}^{k+1}\left(r_{<}, r_{>}\right)= & \left(r_{<}^{2}+r_{>}^{2}\right) f_{\ell}^{k}\left(r_{<}, r_{>}\right)-r_{<} r_{>} \frac{2 \ell}{2 \ell-1} f_{\ell-1}^{k}\left(r_{<}, r_{>}\right) \\
& -r_{<} r_{>} \frac{2(\ell+1)}{2 \ell+3} f_{\ell+1}^{k}\left(r_{<}, r_{>}\right) .
\end{aligned}
$$

Copyright $@$ by SIAM. Unauthorized reproduction of this article is prohibited. 
It is clear from the standard form $r_{12}^{-1}=\sum_{l=0}^{\infty}\left(\ell+\frac{1}{2}\right)^{-1 / 2} \frac{r_{<}^{\ell}}{r_{>}^{\ell+1}} \Phi_{\ell}(\theta)$ that the expansion (6.4) holds for $n=0$. Assuming the expansion (6.4) for $f_{\ell}^{k}\left(r_{<}, r_{>}\right)$and inserting it into (6.9) we have

$$
\begin{aligned}
f_{\ell}^{k+1}\left(r_{<}, r_{>}\right)=r_{<}^{\ell} r_{>}^{-\ell-1}[ & \sum_{j=0}^{k} c_{j}^{k, \ell} r_{<}^{2(k-j+1)} r_{>}^{2 j}+\sum_{j=0}^{k} c_{j}^{k, \ell} r_{<}^{2(k-j)} r_{>}^{2(j+1)} \\
& -\sum_{j=0}^{k} \frac{2 \ell}{2 \ell-1} c_{j}^{k, \ell-1} r_{<}^{2(k-j)} r_{>}^{2(j+1)} \\
& \left.-\sum_{j=0}^{k} \frac{2(\ell+1)}{2 \ell+3} c_{j}^{k, \ell+1} r_{<}^{2(k-j+1)} r_{>}^{2 j}\right]
\end{aligned}
$$

and equating powers of $r_{<}$and $r_{>}$with (6.4) gives (6.5).

We now wish to show that $c_{j}^{k, \ell}$ is of the required form.

Lemma 6.3. The constants $c_{j}^{k, \ell}$ are given by

$$
c_{j}^{k, \ell}=\frac{a_{j}^{k}}{\prod_{m=1}^{k-j}(2 \ell+2 m+1) \prod_{n=1}^{j}(2 \ell-2 n+1)}=: \frac{a_{j}^{k}}{P_{j}^{k}(\ell)},
$$

where

$$
\begin{aligned}
& a_{0}^{k}=\prod_{i=1}^{k}(2 k-2 i+1), \quad \text { and } \\
& a_{j}^{k}=(-1)^{j}\left(\begin{array}{c}
k \\
j
\end{array}\right) a_{0}^{k}, \quad j=1, \ldots, k,
\end{aligned}
$$

with the convention throughout that an empty product takes the value 1.

Proof. We start with the case $j=0, k=0$ which is clearly of the correct form as $a_{0}^{0}=1$. Assuming (6.10) and substituting into (6.5) gives

$$
\frac{a_{j}^{k+1}}{P_{j}^{k+1}(\ell)}=\frac{a_{j}^{k}}{P_{j}^{k}(\ell)}+\frac{a_{j-1}^{k}}{P_{j-1}^{k}(\ell)}-\frac{2 \ell}{2 \ell-1} \frac{a_{j-1}^{k}}{P_{j-1}^{k}(\ell-1)}-\frac{2(\ell+1)}{2 \ell+3} \frac{a_{j}^{k}}{P_{j}^{k}(\ell+1)} .
$$

Which, multiplying by $P_{j}^{k+1}(\ell)$, and simplifying gives

$$
a_{j}^{k+1}=\frac{4 k \ell+2 \ell+2 k+2 j+1}{2 \ell+1} a_{j}^{k}+\frac{-4 k \ell-2 \ell-2 j+1}{2 \ell+1} a_{j-1}^{k} .
$$

For $j=0$, using (6.11) and $a_{-1}^{k}=0$, this gives

$$
a_{0}^{k+1}=\frac{4 k \ell+2 \ell+2 k+1}{2 \ell+1} a_{0}^{k}=(2 k+1) a_{0}^{k}=\prod_{i=1}^{k+1}(2(k+1)-2 i+1),
$$

showing that (6.11) holds for $k+1$. Noting now that (6.12) implies, for $1 \leq j \leq k$,

$$
a_{j-1}^{k}=-\frac{j}{k-j+1} a_{j}^{k}
$$

Copyright $@$ by SIAM. Unauthorized reproduction of this article is prohibited. 
and hence, for $1 \leq j \leq k$, inserting (6.14) into (6.13) gives

$$
a_{j}^{k+1}=\frac{(k+1)(2 \ell+4 k \ell+2 k+1)}{(2 \ell+1)(k-j+1)} a_{j}^{k}=\frac{(k+1)(1+2 k)}{(k-j+1)} a_{j}^{k} .
$$

It remains to show (6.12) holds for $a_{j}^{k+1}$, which is equivalent to

$$
a_{j-1}^{k+1}=-\frac{j}{k-j+2} a_{j}^{k+1}, 1 \leq j \leq k+1 .
$$

Using (6.15) for $a_{j-1}^{k+1}$ and applying (6.14) we get

$$
a_{j-1}^{k+1}=\frac{(k+1)(1+2 k)}{(k-j+2)} a_{j-1}^{k}=-\frac{(k+1)(1+2 k)}{(k-j+2)} \frac{j}{(k-j+1)} a_{j}^{k},
$$

which holds for $1 \leq j \leq k$. Using (6.15) gives (6.16), showing that (6.12) holds for $k+1, j=1, \ldots, k$.

The final case is $j=k+1$. By (6.13) and the fact that $a_{k+1}^{k}=0$ we have

$$
a_{k+1}^{k+1}=a_{k}^{k}\left(\frac{-4 k \ell-2 k-2(k+1)+1}{2 \ell+1}\right)=-(2 k+1) a_{k}^{k} .
$$

Using (6.12) for $j=k$ and (6.11) for $k+1$ this gives

$$
a_{k+1}^{k+1}=(-1)^{k+1}(2 k+1) a_{0}^{k}=(-1)^{k+1} a_{0}^{k+1},
$$

and since $\left(\begin{array}{l}k+1 \\ k+1\end{array}\right)=1$ this gives the result.

Proof of Lemma 6.1. This is a direct consequence of Lemmas 6.2 and 6.3.

In addition, we require an explicit form for $\frac{\partial}{\partial r_{\gamma}} f_{\ell}^{k}\left(r_{<}, r_{>}\right)$.

LEMmA 6.4. The derivatives of $f_{l}^{k}\left(r_{<}, r_{>}\right)$are given by

$$
\begin{gathered}
\frac{\partial}{\partial r_{<}} f_{\ell}^{k}\left(r_{<}, r_{>}\right)=r_{<}^{\ell-1} r_{>}^{-\ell-1} \sum_{j=0}^{k} \frac{a_{j}^{k} r_{<}^{2(k-j)} r_{>}^{2 j}(\ell+2(k-j))}{\prod_{m=1}^{k-j}(2 \ell+2 m+1) \prod_{n=1}^{j}(2 \ell-2 n+1)} \\
\frac{\partial}{\partial r_{>}} f_{\ell}^{k}\left(r_{<}, r_{>}\right)=r_{<}^{\ell} r_{>}^{-\ell-2} \sum_{j=0}^{k} \frac{a_{j}^{k} r_{<}^{2(k-j)} r_{>}^{2 j}(-\ell-1+2 j)}{\prod_{m=1}^{k-j}(2 \ell+2 m+1) \prod_{n=1}^{j}(2 \ell-2 n+1)} .
\end{gathered}
$$

Proof. This follows trivially from differentiating (6.3) term by term.

6.3. Decay of $\boldsymbol{\xi}_{\ell}^{j}\left(\boldsymbol{r}_{<}, \boldsymbol{r}_{>}\right)$. We now wish to use the explicit form of $f_{\ell}^{k}\left(r_{<}, r_{>}\right)$ given in Lemma 6.1 along with the expansion (6.1) to derive the decay rate of the $\xi_{\ell}^{j}\left(r_{<}, r_{>}\right)$in terms of the decay rates of the $f_{\ell}^{k}\left(r_{<}, r_{>}\right)$. Following the ideas of Hill, we write

$$
\left(r_{>}-r_{<}\right)^{j-2 k+1}=r_{>}^{j-2 k+1}\left(1-\frac{r_{<}}{r_{>}}\right)^{j-2 k+1},
$$

and hence, up to a constant, (6.3) shows that the term in the summand of (6.1) may be written as

$$
r_{>}^{j-2 k}\left(1-\frac{r_{\leq}}{r_{>}}\right)^{j-2 k+1}\left(\frac{r_{<}}{r_{>}}\right)^{\ell} \sum_{i=0}^{k} \frac{a_{i}^{k} r_{<}^{2(k-i)} r_{>}^{2 i}}{P_{i}^{k}(\ell)} .
$$

Copyright $@$ by SIAM. Unauthorized reproduction of this article is prohibited. 
We now investigate a general function of the form $h_{p, n}(x)=(1-x)^{p} x^{n}, x \in[0,1]$, $n, p \in \mathbb{R} \backslash\{0\}$, where $x$ will correspond to $r_{<} / r_{>}$, and in particular we would like to find a bound for $h_{p, n}(x)$ in terms of $p$ and $n$. It is clear that

$$
\frac{d}{d x} h_{p, n}(x)=n(1-x)^{p} x^{n-1}-p(1-x)^{p-1} x^{n},
$$

from which it follows that $h_{p, n}(x)$ is maximized at $x=\frac{n}{n+p}$, and the maximum value of $h_{p, n}$ can be bounded from above by $\left(\frac{p}{n}\right)^{p}$.

In our case, we see that $p=j-2 k+1$ and that we may take $n=\ell / m$ with $m>0$. This gives that

$$
\begin{aligned}
\left(r_{>}-r_{<}\right)^{j-2 k+1}\left(\frac{r_{<}}{r_{>}}\right)^{\ell / m} & =r_{>}^{j-2 k+1}\left(1-\frac{r_{<}}{r_{>}}\right)^{j-2 k+1}\left(\frac{r_{<}}{r_{>}}\right)^{\ell / m} \\
& \leq r_{>}^{j-2 k+1}(m(j-2 k+1))^{j-2 k+1} \ell^{-j+2 k-1}
\end{aligned}
$$

which shows that the decay rate in $\ell$ is independent of the choice of $m$. Inserting this bound into (6.1), and using that $1 /(\ell+1 / 2)<1 / \ell$, we see that

$$
\begin{aligned}
\left|\xi_{\ell}^{j}\left(r_{<}, r_{>}\right)\right|^{2} \leq & \ell^{-2 j-3}\left(\frac{1}{j !}\right)^{2} \sum_{k=0}^{\lfloor(j+1) / 2\rfloor} \sum_{k^{\prime}=0}^{\lfloor(j+1) / 2\rfloor} \ell^{2\left(k+k^{\prime}\right)}\left(\begin{array}{c}
j \\
2 k-1
\end{array}\right)\left(\begin{array}{c}
j \\
2 k^{\prime}-1
\end{array}\right) \\
& \times r_{>}^{2 j-2\left(k+k^{\prime}\right)+2}(m(j-2 k+1))^{j-2 k+1}\left(m\left(j-2 k^{\prime}+1\right)\right)^{j-2 k^{\prime}+1} \\
& \times\left|\left(\frac{r_{<}}{r_{>}}\right)^{-2 \ell / m} f_{\ell}^{k}\left(r_{<}, r_{>}\right) f_{\ell}^{k^{\prime}}\left(r_{<}, r_{>}\right)\right|
\end{aligned}
$$

Similarly, differentiating (6.1) and using (6.19) and the analogous version for lower powers of $\left(r_{>}-r_{<}\right)$gives

$$
\begin{gathered}
\left|\frac{\partial}{\partial r_{\gamma}} \xi_{\ell}^{j}\left(r_{<}, r_{>}\right)\right|^{2} \\
\leq \ell^{-2 j-1}\left(\frac{1}{j !}\right)^{2} \sum_{k=0}^{2(j+1) / 2\rfloor} \sum_{k^{\prime}=0}^{\lfloor(j+1) / 2\rfloor} \ell^{2\left(k+k^{\prime}\right)}\left(\begin{array}{c}
j \\
2 k-1
\end{array}\right)\left(\begin{array}{c}
j \\
2 k^{\prime}-1
\end{array}\right) r_{>}^{2 j-2\left(k+k^{\prime}\right)} \\
\times\left[(j-2 k+1)\left(j-2 k^{\prime}+1\right)(m(j-2 k))^{j-2 k}\left(m\left(j-2 k^{\prime}\right)\right)^{j-2 k^{\prime}}\right. \\
\times\left|\left(\frac{r_{<}}{r_{>}}\right)^{-2 \ell / m} f_{\ell}^{k}\left(r_{<}, r_{>}\right) f_{\ell}^{k^{\prime}}\left(r_{<}, r_{>}\right)\right| \\
\times 2 \ell^{-1} r_{>}(j-2 k+1)(m(j-2 k))^{j-2 k}\left(m\left(j-2 k^{\prime}+1\right)\right)^{j-2 k^{\prime}+1} \\
+\left|\left(\frac{r_{<}}{r_{>}}\right)^{-2 \ell / m} f_{\ell}^{k}\left(r_{<}, r_{>}\right) \frac{\partial}{\partial r_{\gamma}} f_{\ell}^{k^{\prime}}\left(r_{<}, r_{>}\right)\right| \\
+\ell^{-2} r_{>}^{2}(m(j-2 k+1))^{j-2 k+1}\left(m\left(j-2 k^{\prime}+1\right)\right)^{j-2 k^{\prime}+1} \\
\left.\times\left|\left(\frac{r_{<}}{r_{>}}\right)^{-2 \ell / m} \frac{\partial}{\partial r_{\gamma}} f_{\ell}^{k}\left(r_{<}, r_{>}\right) \frac{\partial}{\partial r_{\gamma}} f_{\ell}^{k^{\prime}}\left(r_{<}, r_{>}\right)\right|\right] .
\end{gathered}
$$

Copyright $@$ by SIAM. Unauthorized reproduction of this article is prohibited. 
The remaining step is to determine the decay rates of integrals of products of two of $f_{\ell}^{k}\left(r_{<}, r_{>}\right)$and $\frac{\partial}{\partial r_{\gamma}} f_{\ell}^{k}\left(r_{<}, r_{>}\right)$with a general function of $r_{<}$and $r_{>}$, which will later to be chosen to be products of the $A_{j}\left(r_{<}, r_{>}\right)$or their derivatives. Again, by Cauchy-Schwarz it is necessary only to consider integrals of the forms

$$
\int_{0}^{\infty} \int_{0}^{r_{>}} r_{<}^{\alpha} r_{>}^{\beta} g\left(r_{<}, r_{>}\right)\left(\frac{r_{<}}{r_{>}}\right)^{-2 \ell / m}\left|f_{\ell}^{k}\left(r_{<}, r_{>}\right) f_{\ell}^{k^{\prime}}\left(r_{<}, r_{>}\right)\right| d r_{<} d r_{>}
$$

and

$$
\int_{0}^{\infty} \int_{0}^{r_{>}} r_{<}^{\alpha} r_{>}^{\beta} g\left(r_{<}, r_{>}\right)\left(\frac{r_{<}}{r_{>}}\right)^{-2 \ell / m}\left|\frac{\partial}{\partial r_{\gamma}} f_{\ell}^{k}\left(r_{<}, r_{>}\right) \frac{\partial}{\partial r_{\gamma}} f_{\ell}^{k^{\prime}}\left(r_{<}, r_{>}\right)\right| d r_{<} d r_{>},
$$

where $\alpha, \beta \in \mathbb{N} \cup\{0\}$ and $r_{\gamma} \in\left\{r_{<}, r_{>}\right\}$.

6.4. Results on the integrals. The evaluation of (6.22) and (6.23) is obviously highly dependent on the form of $g\left(r_{<}, r_{>}\right)$and, while we have an explicit form for $f_{\ell}^{k}\left(r_{<}, r_{>}\right)$, we do not have an explicit form for $g\left(r_{<}, r_{>}\right)$. We begin by noting the following result on $f_{\ell}^{k}\left(r_{<}, r_{>}\right)$and $\frac{\partial}{\partial r_{\gamma}} f_{\ell}^{k}\left(r_{<}, r_{>}\right)$.

LEMma 6.5. For $m>1$ and $\ell \gg k$, the functions $\left(r_{<} / r_{>}\right)^{-\ell / m} f_{\ell}^{k}\left(r_{<}, r_{>}\right)$and $\left(r_{<} / r_{>}\right)^{-\ell / m} \frac{\partial}{\partial r_{\gamma}} f_{\ell}^{k}\left(r_{<}, r_{>}\right)$for $r_{\gamma} \in\left\{r_{<}, r_{>}\right\}$are strongly peaked on the line $r_{<}=r_{>}$.

Proof. From (6.3), (6.17), and (6.18) we see that a general term in $r_{<}$and $r_{>}$is of the form $r_{<}^{\tilde{\ell}+a} / r_{>}^{\tilde{\ell}+b}$ where $a, b \in\{-2, \ldots, 2 k\}$ and $\tilde{\ell}=(m-1) \ell / m>0$. Rewriting this and using that $r_{<}=r_{>}-x$ where $x \in\left[0, r_{>}\right]$we have that

$$
\frac{r_{<}^{\tilde{\ell}+a}}{r_{>}^{\tilde{\ell}+b}}=r_{>}^{a-b}\left(\frac{r_{<}}{r_{>}}\right)^{\tilde{\ell}+a}=r_{>}^{a-b}\left(1-\frac{x}{r_{>}}\right)^{\tilde{\ell}+b} .
$$

For $\ell \gg k$ we have $\tilde{\ell}+b \gg 1$ and this expression is strongly peaked when $x=0$, giving the result.

For suitably smooth $g\left(r_{<}, r_{>}\right)$, this result implies that it is sensible to Taylor expand $g\left(r_{<}, r_{>}\right)$around $r_{<}=r_{>}$.

LEMma 6.6. Let $g\left(\cdot, r_{>}\right) \in C^{N}(\mathbb{R})$ be such that $\left|g^{(n)}\left(s, r_{>}\right)\right| \leq \tilde{g}\left(r_{>}\right)$for all $s \in \mathbb{R}_{+}$and for $n=0, \ldots, N$. Then, for $M<N, p>0$, and $f\left(r_{<}, r_{>}\right)$an arbitrary function,

$$
\begin{aligned}
\int_{0}^{r_{>}} g\left(r_{<}, r_{>}\right)\left|f\left(r_{<}, r_{>}\right)\right| d r_{<}= & \sum_{m=0}^{M} g^{(m)}\left(r_{>}, r_{>}\right) \int_{0}^{r_{>}} \frac{\left(r_{<}-r_{>}\right)^{m}}{m !}\left|f\left(r_{<}, r_{>}\right)\right| d r_{<} \\
& +\sum_{n=M+1}^{N} \mathcal{O}\left(\ell^{-n}\right) \tilde{g}\left(r_{>}\right) \int_{0}^{r_{>}} r_{<}^{n}\left(\frac{r_{<}}{r_{>}}\right)^{-\ell / p}\left|f\left(r_{<}, r_{>}\right)\right| d r_{<} .
\end{aligned}
$$

Proof. We begin by noting that we may Taylor expand $g\left(r_{<}, r_{>}\right)$in $r_{<}$about $r_{>}$ and, since $g^{(N)}$ is continuous, we may use the Lagrange form of the remainder, i.e.,

$$
g\left(r_{<}, r_{>}\right)=\sum_{n=0}^{N-1} \frac{\left(r_{<}-r_{>}\right)^{n}}{n !} g^{(n)}\left(r_{>}, r_{>}\right)+\frac{\left(r_{<}-r_{>}\right)^{N}}{N !} g^{(N)}\left(s, r_{>}\right)
$$

for some point $s \in\left(r_{<}, r_{>}\right)$. It is clear that the first $M$ terms of this expansion contribute the terms in the sum in (6.24). We now note that, in a similar way to the 
derivation of (6.19),

$$
\left(r_{<}-r_{>}\right)^{n}\left(\frac{r_{<}}{r_{>}}\right)^{\ell / p} \leq c r_{>}^{n} \ell^{-n}
$$

for any $p>0$ and for some $c>0$ (which depends on $p$ ). Hence, since $\left|g^{(n)}\left(r, r_{>}\right)\right| \leq$ $\tilde{g}\left(r_{>}\right)$for all $r$, the result follows.

Applying this result to the integrals (6.22) and (6.23), we find the general integrals are now of the form

$$
\int_{0}^{\infty} \int_{0}^{r_{>}} r_{<}^{\alpha} r_{>}^{\beta} g\left(r_{>}\right)\left(\frac{r_{<}}{r_{>}}\right)^{-2 \ell / m}\left|f_{\ell}^{k}\left(r_{<}, r_{>}\right) f_{\ell}^{k^{\prime}}\left(r_{<}, r_{>}\right)\right| d r_{<} d r_{>}
$$

and

$$
\int_{0}^{\infty} \int_{0}^{r_{>}} r_{<}^{\alpha} r_{>}^{\beta} g\left(r_{>}\right)\left(\frac{r_{<}}{r_{>}}\right)^{-2 \ell / m}\left|\frac{\partial}{\partial r_{\gamma}} f_{\ell}^{k}\left(r_{<}, r_{>}\right) \frac{\partial}{\partial r_{\gamma}} f_{\ell}^{k^{\prime}}\left(r_{<}, r_{>}\right)\right| d r_{<} d r_{>}
$$

The advantage of this form of the integrals is that, since the unknown function $g$ is no longer dependent on $r_{<}$, we may explicitly evaluate the $r_{<}$integral. We now move on to discuss the decay rates of integrals of the types (6.25) and (6.26).

6.5. Decay rates of the integrals. From Hill's result we expect the norms (5.2) and (5.3) to decay at least as $\mathcal{O}\left(\ell^{-4}\right)$. In fact, since the norm in (5.2) is the $L^{2}$ norm and that in (5.3) is a higher Sobolev norm, we expect the slowest decay to come from the energy norm. Recall that we have factors of $\ell^{-2 j-3+2\left(k+k^{\prime}\right)}$, $\ell^{-2 j-1+2\left(k+k^{\prime}\right)}$, and $\ell^{-2 j-3+2\left(k+k^{\prime}\right)}$ for terms involving $f_{\ell}^{k}\left(r_{<}, r_{>}\right) f_{\ell}^{k^{\prime}}\left(r_{<}, r_{>}\right)$from (6.20), $f_{\ell}^{k}\left(r_{<}, r_{>}\right) f_{\ell}^{k^{\prime}}\left(r_{<}, r_{>}\right)$from (6.21), and $\frac{\partial}{\partial r_{\alpha}} f_{\ell}^{k}\left(r_{<}, r_{>}\right) \frac{\partial}{\partial r_{\alpha}} f_{\ell}^{k^{\prime}}\left(r_{<}, r_{>}\right)$from (6.21), respectively.

The main aim of this section is to remove the dependence on $k$ and $k^{\prime}$ from the decay rates of $\left|\xi_{\ell}^{j}\left(r_{<}, r_{>}\right)\right|^{2}$ and $\left|\frac{\partial}{\partial r_{\gamma}} \xi_{\ell}^{j}\left(r_{<}, r_{>}\right)\right|$. We begin by finding the $k$-dependence of integrals of the forms (6.25) and (6.26).

LEMMA 6.7. For $k, k^{\prime} \geq 1, \alpha \in \mathbb{N} \cup\{0\}, r_{\gamma} \in\left\{r_{<}, r_{>}\right\}$, and $m>1$, let $\tilde{\ell}:=$ $(m-1) \ell / m$, then

$$
\begin{aligned}
& \int_{0}^{r_{>}} r_{<}^{\alpha}\left(\frac{r_{<}}{r_{>}}\right)^{-2 \ell / m}\left|f_{\ell}^{k}\left(r_{<}, r_{>}\right) f_{\ell}^{k^{\prime}}\left(r_{<}, r_{>}\right)\right| d r_{<} \\
& =\frac{r_{>}^{2\left(k+k^{\prime}\right)+\alpha-1}}{P^{k}(\ell) P^{k^{\prime}}(\ell) Q^{k, k^{\prime}}(\tilde{\ell} ; \alpha)} \sum_{j=0}^{k} \sum_{j^{\prime}=0}^{k^{\prime}} a_{j}^{k} a_{j^{\prime}}^{k^{\prime}} R_{j, j^{\prime}}^{k, k^{\prime}}(\ell, \tilde{\ell} ; \alpha)
\end{aligned}
$$

$$
\begin{aligned}
\int_{0}^{r_{>}} & r_{<}^{\alpha}\left(\frac{r_{<}}{r_{>}}\right)^{-2 \ell / m}\left|\frac{\partial}{\partial r_{<}} f_{\ell}^{k}\left(r_{<}, r_{>}\right) \frac{\partial}{\partial r_{<}} f_{\ell}^{k^{\prime}}\left(r_{<}, r_{>}\right)\right| d r_{<} \\
= & \frac{r_{>}^{2\left(k+k^{\prime}\right)+\alpha-3}}{P^{k}(\ell) P^{k^{\prime}}(\ell) Q^{k, k^{\prime}}(\tilde{\ell}-1 ; \alpha)} \\
& \times \sum_{j=0}^{k} \sum_{j^{\prime}=0}^{k^{\prime}} a_{j}^{k} a_{j^{\prime}}^{k^{\prime}} R_{j, j^{\prime}}^{k, k^{\prime}}(\ell, \tilde{\ell}-1 ; \alpha)(\ell+2(k-j))\left(\ell+2\left(k^{\prime}-j^{\prime}\right)\right), \quad \text { and }
\end{aligned}
$$

Copyright (C) by SIAM. Unauthorized reproduction of this article is prohibited. 


$$
\begin{aligned}
\int_{0}^{r_{>}} r_{<}^{\alpha} & \left(\frac{r_{<}}{r_{>}}\right)^{-2 \ell / m}\left|\frac{\partial}{\partial r_{>}} f_{\ell}^{k}\left(r_{<}, r_{>}\right) \frac{\partial}{\partial r_{>}} f_{\ell}^{k^{\prime}}\left(r_{<}, r_{>}\right)\right| d r_{<} \\
& =\frac{r_{>}^{2\left(k+k^{\prime}\right)+\alpha-3}}{P^{k}(\ell) P^{k^{\prime}}(\ell) Q^{k, k^{\prime}}(\tilde{\ell} ; \alpha)} \sum_{j=0}^{k} \sum_{j^{\prime}=0}^{k^{\prime}} a_{j}^{k} a_{j^{\prime}}^{k^{\prime}} R_{j, j^{\prime}}^{k, k^{\prime}}(\ell, \tilde{\ell} ; \alpha)(\ell-2 j)\left(\ell-2 j^{\prime}\right),
\end{aligned}
$$

where $P^{k}(\ell)$ is a polynomial in $\ell$ of degree $2 k$, given explicitly in $(6.30), Q^{k, k^{\prime}}(\tilde{\ell} ; \alpha)$ is a polynomial in $\tilde{\ell}$ of degree $k+k^{\prime}+1$ with coefficients parameterized by $\alpha$ and is given explicitly in (6.32). The remaining term $R_{j, j^{\prime}}^{k, k^{\prime}}(\ell, \tilde{\ell} ; \alpha)$ is the product of a polynomial in $\ell$ with a polynomial in $\tilde{\ell}$ (both of degree $k+k^{\prime}$ ), with coefficients parameterized by $\alpha$ and is given explicitly in (6.33).

Proof. We prove the result for $f_{\ell}^{k}\left(r_{<}, r_{>}\right)$, the proofs for $\frac{\partial}{\partial r_{\gamma}} f_{\ell}^{k}\left(r_{<}, r_{>}\right)$are analogous. By (6.3) we have that

$$
\left(\frac{r_{<}}{r_{>}}\right)^{-2 \ell / m} f_{\ell}^{k}\left(r_{<}, r_{>}\right) f_{\ell}^{k^{\prime}}\left(r_{<}, r_{>}\right)=\frac{r_{<}^{2 \tilde{\ell}}}{r_{>}^{2 \tilde{\ell}+2}} \sum_{j=0}^{k} \sum_{j^{\prime}=0}^{k^{\prime}} \frac{a_{j}^{k} a_{j^{\prime}}^{k^{\prime}} r_{<}^{2\left(k+k^{\prime}-j-j^{\prime}\right)} r_{>}^{2\left(j+j^{\prime}\right)}}{P_{j}^{k}(\ell) P_{j^{\prime}}^{k^{\prime}}(\ell)},
$$

and hence

$$
\begin{aligned}
& \int_{0}^{r_{>}} r_{<}^{\alpha}\left(\frac{r_{<}}{r_{>}}\right)^{-2 \ell / m} f_{\ell}^{k}\left(r_{<,} r_{>}\right) f_{\ell}^{k^{\prime}}\left(r_{<}, r_{>}\right) d r_{<} \\
& =\int_{0}^{r>} r_{<}^{2 \tilde{\ell}+\alpha} r_{>}^{-2 \tilde{\ell}-2} \sum_{j=0}^{k} \sum_{j^{\prime}=0}^{k^{\prime}} \frac{a_{j}^{k} a_{j^{\prime}}^{k^{\prime}} r_{<}^{2\left(k+k^{\prime}-j-j^{\prime}\right)} r_{>}^{2\left(j+j^{\prime}\right)}}{P_{j}^{k}(\ell) P_{j^{\prime}}^{k^{\prime}}(\ell)} d r_{<} \\
& =r_{>}^{2\left(k+k^{\prime}\right)+\alpha-1} \sum_{j=0}^{k} \sum_{j^{\prime}=0}^{k^{\prime}} \frac{a_{j}^{k} a_{j^{\prime}}^{k^{\prime}}}{P_{j}^{k}(\ell) P_{j^{\prime}}^{k^{\prime}}(\ell)\left(2 \tilde{\ell}+2\left(k+k^{\prime}-j-j^{\prime}\right)+\alpha+1\right)} .
\end{aligned}
$$

We now define the polynomial

$$
P^{k}(\ell):=\prod_{m=1}^{k}(2 \ell+2 m+1) \prod_{n=1}^{k}(2 \ell-2 n+1),
$$

which is the product of all distinct terms in the denominators of (6.3) over all $j$. We also note that the terms given by the integration are

$$
\left\{\left(2 \tilde{\ell}+2\left(k+k^{\prime}-j-j^{\prime}\right)+\alpha+1\right)\right\}_{j+j^{\prime}=0}^{k+k^{\prime}}=\{(2 \tilde{\ell}+2 i+\alpha-1)\}_{i=1}^{k+k^{\prime}+1},
$$

and we therefore define

$$
Q^{k, k^{\prime}}(\tilde{\ell} ; \alpha)=\prod_{i=1}^{k+k^{\prime}+1}(2 \tilde{\ell}+2 i+\alpha-1)
$$

It follows that

$$
\begin{aligned}
\int_{0}^{r_{>}} r_{<}^{\alpha}\left(\frac{r_{<}}{r_{>}}\right)^{-2 \ell / m}\left|f_{\ell}^{k}\left(r_{<}, r_{>}\right) f_{\ell}^{k^{\prime}}\left(r_{<}, r_{>}\right)\right| d r_{<} \\
=\frac{r_{>}^{2\left(k+k^{\prime}\right)+\alpha-1}}{P^{k}(\ell) P^{k^{\prime}}(\ell) Q^{k, k^{\prime}}(\tilde{\ell} ; \alpha)}\left|\sum_{j=0}^{k} \sum_{j^{\prime}=0}^{k^{\prime}} a_{j}^{k} a_{j^{\prime}}^{k^{\prime}} R_{j, j^{\prime}}^{k, k^{\prime}}(\ell, \tilde{\ell} ; \alpha)\right|,
\end{aligned}
$$

Copyright (c) by SIAM. Unauthorized reproduction of this article is prohibited. 
where

$$
\begin{aligned}
R_{j, j^{\prime}}^{k, k^{\prime}}(\ell, \tilde{\ell} ; \alpha)= & \prod_{m=k-j+1}^{k}(2 \ell+2 m+1) \prod_{n=j+1}^{k}(2 \ell-2 n+1) \\
& \times \prod_{\substack{m^{\prime}=k^{\prime}-j^{\prime}+1 \\
k+k^{\prime}+1}}^{k}\left(2 \ell+2 m^{\prime}+1\right) \prod_{n^{\prime}=j^{\prime}+1}^{k^{\prime}}\left(2 \ell-2 n^{\prime}+1\right) \\
& \times \prod_{\substack{i=1 \\
i \neq\left(k+k^{\prime}-j-j^{\prime}+1\right)}}^{k}(2 i \ell+\alpha-1) .
\end{aligned}
$$

For $\frac{\partial}{\partial r_{>}} f_{\ell}^{k}\left(r_{<}, r_{>}\right)$the power of $r_{<}$is unchanged, leading to the same coefficients from the integration but a reduced power of $r_{>}$. The extra two terms in $\ell$ come from the initial differentiation as given in (6.18). For $\frac{\partial}{\partial r_{<}} f_{\ell}^{k}\left(r_{<}, r_{>}\right)$the initial power of $r_{<}$ is reduced by two, and we once again gain two factors from the initial differentiation as shown in (6.17).

The results of Lemma 6.7 show (noting that $\mathcal{O}(\tilde{\ell})=\mathcal{O}(\ell)$ ) that the integral (6.27) is of order $\ell^{-\left(k+k^{\prime}\right)-1}$ and the integrals (6.28) and (6.29) are of order $\ell^{-\left(k+k^{\prime}\right)+1}$. However, this is not strong enough to counter the $\ell^{2\left(k+k^{\prime}\right)}$ in the summands of (6.20) and (6.21). We would therefore like to show that the summation over $j$ and $j^{\prime}$ in (6.27)-(6.29) causes an increase in order.

The first step is to note that $R_{j, j^{\prime}}^{k, k^{\prime}}(\ell, \tilde{\ell} ; \alpha)$ and the $(\ell+\beta)$ terms in these sums contribute powers of $j$ and $j^{\prime}$. The terms in (6.31), i.e., those in the final product of $R_{j, j^{\prime}}^{k, k^{\prime}}(\ell, \tilde{\ell} ; \alpha)$ in (6.33), may be written in the form $\left(2 \tilde{\ell}+\gamma_{i}-\left(j+j^{\prime}\right)\right)$ where the $\gamma_{i}$ depend on $k, k^{\prime}$, and $\alpha$ but are independent of $j, j^{\prime}$, and $\ell$. There are $k+k^{\prime}$ of these terms, and this product may be expanded as

$$
\begin{aligned}
\prod_{\substack{i=1 \\
i \neq\left(k+k^{\prime}-j-j^{\prime}+1\right)}}^{k+k^{\prime}+1}(2 \tilde{\ell}+2 i+\alpha-1) & =\sum_{n=0}^{k+k^{\prime}} c_{n} \tilde{\ell}^{n}\left(j+j^{\prime}\right)^{k+k^{\prime}-n} \\
& =\sum_{n=0}^{k+k^{\prime}} \tilde{\ell}^{n} \sum_{m=0}^{k+k^{\prime}-n} d_{n, m} j^{m} j^{\prime k+k^{\prime}-n-m}
\end{aligned}
$$

where $c_{n}$ and $d_{n, m}$ depend on $k, k^{\prime}$, and $\alpha$. Similarly, we see that the extra two terms in (6.28) and (6.29) lead to sums of the form

$$
\sum_{n=0}^{k+k^{\prime}} c_{n} \tilde{\ell}^{n}\left(j+j^{\prime}\right)^{k+k^{\prime}-n} j^{x} j^{\prime y} \ell^{2-x-y}=\sum_{n=0}^{k+k^{\prime}} \tilde{\ell}^{n} \ell^{2-x-y} \sum_{m=0}^{k+k^{\prime}-n} d_{n, m} j^{m+x} j^{\prime k+k^{\prime}-n+y},
$$

where $x, y \in\{0,1\}$.

From these expansions and the explicit form of $R_{j, j^{\prime}}^{k, k^{\prime}}(\ell, \tilde{\ell} ; \alpha)$ given in $(6.33)$ we see that, for fixed $n$ and $m$, the double sum over $j$ and $j^{\prime}$ in (6.27) is, up to a constant, 
of the form

$$
\begin{aligned}
& \sum_{j=0}^{k} \sum_{j^{\prime}=0}^{k^{\prime}} a_{j}^{k} a_{j^{\prime}}^{k^{\prime}} \tilde{\ell}^{n} j^{m} j^{\prime k+k^{\prime}-n-m} \prod_{\mu=k-j+1}^{k}(2 \ell+2 \mu+1) \prod_{\nu=j+1}^{k}(2 \ell-2 \nu+1) \\
& \times \prod_{\mu^{\prime}=k^{\prime}-j^{\prime}+1}^{k^{\prime}}\left(2 \ell+2 \mu^{\prime}+1\right) \prod_{\nu^{\prime}=j^{\prime}+1}^{k}\left(2 \ell-2 \nu^{\prime}+1\right) . \\
& =\tilde{\ell}^{n} \sum_{j=0}^{k} a_{j}^{k} j^{m} \prod_{\mu=k-j+1}^{k}(2 \ell+2 \mu+1) \prod_{\nu=j+1}^{k}(2 \ell-2 \nu+1) \\
& \quad \times \sum_{j^{\prime}=0}^{k^{\prime}} a_{j^{\prime}}^{k^{\prime}} j^{\prime k+k^{\prime}-n-m} \prod_{\mu^{\prime}=k^{\prime}-j^{\prime}+1}^{k^{\prime}}\left(2 \ell+2 \mu^{\prime}+1\right) \prod_{\nu^{\prime}=j^{\prime}+1}^{k^{\prime}}\left(2 \ell-2 \nu^{\prime}+1\right) .
\end{aligned}
$$

The sums in (6.28) and (6.29) are of the same form but the sum over $j$ contains either an extra power of $\ell$ or an extra power of $j$ and analogously for $j^{\prime}$. We now wish to show.

Lemma 6.8. For $a_{j}^{k}=(-1)^{j}\left(\begin{array}{c}k \\ j\end{array}\right) a_{0}^{k}$, and $p \in \mathbb{N} \cup\{0\}$, the sum

$$
S_{k, p}:=\sum_{j=0}^{k} a_{j}^{k} j^{p} \prod_{m=k-j+1}^{k}(2 \ell+2 m+1) \prod_{n=j+1}^{k}(2 \ell-2 n+1)
$$

is $\mathcal{O}\left(\ell^{p}\right)$.

Proof. We first note that if $p \geq k$, then the result is trivial as the highest order of $\ell$ possible is $k$. Hence we need only consider $0 \leq p<k$ and begin by dividing through by $2^{k}$, letting $\alpha:=\left(\ell+\frac{1}{2}\right)$, using the explicit form (6.12) for $a_{j}^{k}$, and rearranging to give

$$
c S_{k, p}=\sum_{j=0}^{k}(-1)^{j}\left(\begin{array}{l}
k \\
j
\end{array}\right) j^{p} \prod_{m=k-j+1}^{k}(\alpha+m) \prod_{n=j+1}^{k}(\alpha-n),
$$

for some constant c.

Recall the Gamma functions [1], which satisfy

$$
\Gamma(x)=(x-1) \Gamma(x-1), x \in \mathbb{R} .
$$

This gives that

$$
\prod_{m=k-j+1}^{k}(\alpha+m)=\frac{\Gamma(\alpha+k+1)}{\Gamma(\alpha+k+1-j)}, \prod_{n=j+1}^{k}(\alpha-n)=(-1)^{k-j} \frac{\Gamma(k+1-\alpha)}{\Gamma(j+1-\alpha)},
$$

and the standard form of the binomial coefficient is

$$
\left(\begin{array}{c}
k \\
j
\end{array}\right)=\frac{\Gamma(k+1)}{\Gamma(j+1) \Gamma(k-j+1)}
$$

It follows that

$$
c S_{k, p}=(-1)^{k} \sum_{j=0}^{k} j^{p} \frac{\Gamma(k+1)}{\Gamma(j+1) \Gamma(k-j)} \frac{\Gamma(\alpha+k+1)}{\Gamma(\alpha+k+1-j)} \frac{\Gamma(k+1-\alpha)}{\Gamma(j+1-\alpha)} .
$$

Copyright (c) by SIAM. Unauthorized reproduction of this article is prohibited. 
We will prove the cases $0 \leq p<k$ by induction on $p$ and begin with the case $p=0$. Using the identity

$$
\frac{\Gamma(z)}{\Gamma(w)}=\Gamma(z-w+1) \frac{\Gamma(z)}{\Gamma(z-w+1) \Gamma(w)}=(z-w) !\left(\begin{array}{c}
z-1 \\
z-w
\end{array}\right)
$$

and the Vandermonde identity [1] for the sum of binomial coefficients

$$
\sum_{j=0}^{k}\left(\begin{array}{l}
n \\
j
\end{array}\right)\left(\begin{array}{c}
m \\
k-j
\end{array}\right)=\left(\begin{array}{c}
n+m \\
k
\end{array}\right)
$$

gives

$$
\frac{\Gamma(\alpha+k+1)}{\Gamma(\alpha+k+1-j)}=j !\left(\begin{array}{c}
\alpha+k \\
j
\end{array}\right) \text { and } \frac{\Gamma(k+1-\alpha)}{\Gamma(j+1-\alpha)}=(k-j) !\left(\begin{array}{c}
k-\alpha \\
k-j
\end{array}\right) .
$$

Hence, using (6.39) and (6.37),

$$
\begin{aligned}
c S_{k, 0} & =(-1)^{k} \Gamma(k+1) \sum_{j=0}^{k}\left(\begin{array}{c}
\alpha+k \\
j
\end{array}\right)\left(\begin{array}{c}
k-\alpha \\
k-j
\end{array}\right)=(-1)^{k} \Gamma(k+1)\left(\begin{array}{c}
2 k \\
k
\end{array}\right) \\
& =(-1)^{k} \frac{\Gamma(2 k+1)}{\Gamma(k+1)}=\mathcal{O}(1),
\end{aligned}
$$

which shows the result holds for $p=0$. For $0<p<k$ we consider the sum

$$
\begin{aligned}
T_{k, p}:=(-1)^{k} \sum_{j=0}^{k} j(j-1) & \ldots(j-(p-1)) \frac{\Gamma(k+1)}{\Gamma(j+1) \Gamma(k-j+1)} \\
& \times \frac{\Gamma(\alpha+k+1)}{\Gamma(\alpha+k+1-j)} \frac{\Gamma(k+1-\alpha)}{\Gamma(j+1-\alpha)},
\end{aligned}
$$

which is clearly zero when $j \in\{0, \ldots,(p-1)\}$ and thus

$$
\begin{aligned}
T_{k, p}= & (-1)^{k} \sum_{j=p}^{k} \frac{\Gamma(j+1)}{\Gamma(j-p+1)} \frac{\Gamma(k+1)}{\Gamma(j+1) \Gamma(k-j+1)} \frac{\Gamma(\alpha+k+1)}{\Gamma(\alpha+k+1-j)} \frac{\Gamma(k+1-\alpha)}{\Gamma(j+1-\alpha)} \\
= & (-1)^{k} \sum_{j=0}^{k-p} \frac{\Gamma(k+1) \Gamma(\alpha+k+1)}{\Gamma(\alpha+k-p+1)} \frac{\Gamma(\alpha+k-p+1)}{\Gamma(j+1) \Gamma(\alpha+k-p-j+1)} \\
& \times \frac{\Gamma(k-\alpha+1)}{\Gamma(k-j-p+1) \Gamma(p+j-\alpha+1)},
\end{aligned}
$$

We now apply (6.37) to the last two terms, and apply (6.39) to obtain

$$
T_{k, p}=(-1)^{k} \Gamma(k+1) \frac{\Gamma(\alpha+k+1)}{\Gamma(\alpha+k-p+1)}\left(\begin{array}{c}
2 k-p \\
k-p
\end{array}\right) .
$$

It is clear from (6.36) that $\Gamma(\alpha+k+1) / \Gamma(\alpha+k-p+1)$ is $\mathcal{O}\left(\alpha^{p}\right)$, which is equivalent to $\mathcal{O}\left(\ell^{p}\right)$. Finally noting that $j(j-1) \ldots(j-(p-1))=j^{p}+\sum_{i=0}^{p-1} c_{i} j^{i}$ and using the inductive hypothesis gives the result. 
We are now in a position to determine the decay rates of the integrals (6.27)(6.29).

Lemma 6.9. Let $m>1$, then, for $r_{\gamma} \in\left\{r_{<}, r_{>}\right\}$,

$$
\begin{array}{r}
\int_{0}^{r_{>}} r_{<}^{\alpha}\left(\frac{r_{<}}{r_{>}}\right)^{-2 \ell / m}\left|f_{\ell}^{k}\left(r_{<}, r_{>}\right) f_{\ell}^{k^{\prime}}\left(r_{<}, r_{>}\right)\right| d r_{<} \\
=r_{>}^{2\left(k+k^{\prime}\right)+\alpha-1} \mathcal{O}\left(\ell^{-2\left(k+k^{\prime}\right)-1}\right), \\
\int_{0}^{r_{>}} r_{<}^{\alpha}\left(\frac{r_{<}}{r_{>}}\right)^{-2 \ell / m}\left|\frac{\partial}{\partial r_{\gamma}} f_{\ell}^{k}\left(r_{<}, r_{>}\right) \frac{\partial}{\partial r_{\gamma}} f_{\ell}^{k^{\prime}}\left(r_{<}, r_{>}\right)\right| d r_{<}=r_{>}^{2\left(k+k^{\prime}\right)+\alpha-3} \mathcal{O}\left(\ell^{-2\left(k+k^{\prime}\right)+1}\right) .
\end{array}
$$

Proof. Lemma 6.8 and (6.35) show that the sums in (6.34) are overall of order $\mathcal{O}\left(\ell^{k+k}\right)$ and the analogous sums for the derivative cases given in (6.28) and (6.29) are of the form $\mathcal{O}\left(\ell^{k+k^{\prime}+2}\right)$. Combining this order with that of the polynomials outside the sum in (6.27)-(6.29), which are of order $\ell^{-3\left(k+k^{\prime}\right)-1}$, gives the result for the order. The result for the power of $r>$ follow directly from the results of Lemma 6.7.

By (6.20) and (6.21), along with Cauchy-Schwarz, we obtain

$$
\begin{gathered}
\iint r_{<}^{\alpha} r_{<}^{\beta} g\left(r_{>}\right)\left|\xi_{\ell}^{j}\left(r_{<,}, r_{>}\right)\right|^{2} d r_{<} d r_{>} \leq \ell^{-2 j-3} \sum_{k=0}^{\lfloor(j+1) / 2\rfloor} \sum_{k^{\prime}=0}^{\lfloor(j+1) / 2\rfloor} \ell^{2\left(k+k^{\prime}\right)} c_{j, k, k^{\prime}, m}^{(1)} \\
\times \iint r_{<}^{\alpha} r_{>}^{2 j-2\left(k+k^{\prime}\right)+\beta+2} g\left(r_{>}\right)\left(\frac{r_{<}}{r_{>}}\right)^{-2 \ell / m}\left|f_{\ell}^{k}\left(r_{<}, r_{>}\right) f_{\ell}^{k^{\prime}}\left(r_{<}, r_{>}\right)\right| d r_{<} d r_{>}, \\
\iint r_{<}^{\alpha} r_{>}^{\beta} g\left(r_{>}\right)\left|\frac{\partial}{\partial r_{\gamma}} \xi_{\ell}^{j}\left(r_{<,} r_{>}\right)\right|^{2} d r_{<} d r_{>} \leq \ell^{-2 j-1} \sum_{k=0}^{\lfloor(j+1) / 2\rfloor} \sum_{k^{\prime}=0}^{\lfloor(j+1) / 2\rfloor} \ell^{2\left(k+k^{\prime}\right)} \\
\times\left[c_{j, k, k^{\prime}, m}^{(2)} \iint r_{<}^{\alpha} r_{>}^{2 j-2\left(k+k^{\prime}\right)+\beta}\left(\frac{r_{<}}{r_{>}}\right)^{-2 \ell / m}\left|f_{\ell}^{k}\left(r_{<}, r_{>}\right) f_{\ell}^{k^{\prime}}\left(r_{<}, r_{>}\right)\right| d r_{<} d r_{>}\right. \\
+\ell^{-2} c_{j, k, k^{\prime}, m}^{(3)} \iint r_{<}^{\alpha} r_{>}^{2 j-2\left(k+k^{\prime}\right)+2+\beta}\left(\frac{r_{<}}{r_{>}}\right)^{-2 \ell / m} \\
\left.\times\left|\frac{\partial}{\partial r_{\gamma}} f_{\ell}^{k}\left(r_{<}, r_{>}\right) \frac{\partial}{\partial r_{\gamma}} f_{\ell}^{k^{\prime}}\left(r_{<}, r_{>}\right)\right| d r_{<} d r_{>}\right],
\end{gathered}
$$

for some constants $c_{j, k, k^{\prime}, m}^{(i)}, i=1,2,3$.

This holds for any $m>1$ and the bounds in Lemma 6.9 are independent of the choice of $m$ and so, noting that $\mathcal{O}(\ell)=\mathcal{O}(\tilde{\ell})$, we find

$$
\iint r_{<}^{\alpha} r_{>}^{\beta} g\left(r_{>}\right)\left|\frac{\partial}{\partial r_{\gamma}} \xi_{\ell}^{j}\left(r_{<}, r_{>}\right)\right|^{2} d r_{<} d r_{>}=\mathcal{O}\left(\ell^{-2 j-2}\right) \int r_{>}^{2 j-1+\alpha+\beta} g\left(r_{>}\right) d r_{>}
$$

These bounds lead to the following result about the decay rates of the norms of $\psi_{\ell}\left(r_{<}, r_{>}\right)$and $\frac{\partial}{\partial r_{\gamma}} \psi_{\ell}\left(r_{<}, r_{>}\right)$given in (5.7) and (5.8).

Copyright $@$ by SIAM. Unauthorized reproduction of this article is prohibited. 
Corollary 6.10. Let $g\left(\cdot, r_{>}\right) \in C^{N}(\mathbb{R})$ be such that $\left|g^{(n)}\left(s, r_{>}\right)\right| \leq \tilde{g}\left(r_{>}\right)$for some $\tilde{g}\left(r_{>}\right)$, all $s \in \mathbb{R}$ and $n=0, \ldots, N$. Then, for any $M<N$, and $r_{\gamma} \in\left\{r_{<}, r_{>}\right\}$,

$$
\begin{gathered}
\left\|g\left(r_{<}, r_{>}\right) \xi_{\ell}^{j}\left(r_{<}, r_{>}\right)\right\|_{a, b}^{2} \\
=\mathcal{O}\left(\ell^{-2 j-4}\right)\left[\sum_{m=0}^{M} \int_{0}^{\infty} r_{>}^{2 j+1+a+b} \frac{\left(r_{<}-r_{>}\right)^{m}}{m !}\left[\frac{\partial^{m}}{\partial r_{<}^{m}}\left|g\left(r_{<}, r_{>}\right)\right|^{2}\right]_{r_{<}=r_{>}} d r_{>}\right. \\
\left.\quad+\sum_{n=M+1}^{N} \mathcal{O}\left(\ell^{-n}\right) \int_{0}^{\infty} r_{>}^{2 j+1+a+b+n} \tilde{g}\left(r_{>}\right) d r_{>}\right] \\
\text {and } \begin{aligned}
&\left\|g\left(r_{<}, r_{>}\right) \frac{\partial}{\partial r_{\gamma}} \xi_{\ell}^{j}\left(r_{<}, r_{>}\right)\right\|_{a, b}^{2} \\
&=\mathcal{O}\left(\ell^{-2 j-2}\right) {\left[\sum_{m=0}^{M} \int_{0}^{\infty} r_{>}^{2 j-1+a+b} \frac{\left(r_{<}-r_{>}\right)^{m}}{m !}\left[\frac{\partial^{m}}{\partial r_{<}^{m}}\left|g\left(r_{<}, r_{>}\right)\right|^{2}\right]_{r_{<}=r_{>}} d r_{>}\right.} \\
&\left.+\sum_{n=M+1}^{N} \mathcal{O}\left(\ell^{-n}\right) \int_{0}^{\infty} r_{>}^{2 j-1+a+b+n} \tilde{g}\left(r_{>}\right) d r_{>}\right] .
\end{aligned}
\end{gathered}
$$

Proof. We first insert the expansions given in (6.20) and (6.21), with $m=2$ into $\left\|g\left(r_{<}, r_{>}\right) \xi_{\ell}^{j}\left(r_{<}, r_{>}\right)\right\|_{a, b}^{2}$ and $\left\|g\left(r_{<}, r_{>}\right) \frac{\partial}{\partial r_{\gamma}} \xi_{\ell}^{j}\left(r_{<}, r_{>}\right)\right\|_{a, b}^{2}$, respectively.

Next we apply Lemma 6.6 with $p=2, g\left(r_{<}, r_{>}\right)=\left|g\left(r_{<}, r_{>}\right)\right|^{2}$, and $f\left(r_{<}, r_{>}\right)=$ $r_{<}^{a} r_{>}^{b} f_{\ell}^{k}\left(r_{<}, r_{>}\right) f_{\ell}^{k^{\prime}}\left(r_{<}, r_{>}\right)$or $r_{<}^{a} r_{>}^{b} \frac{\partial}{\partial r_{\gamma}} f_{\ell}^{k}\left(r_{<}, r_{>}\right) \frac{\partial}{\partial r_{\gamma}} f_{\ell}^{k^{\prime}}\left(r_{<}, r_{>}\right)$to each of the $r_{<}$integrals, resulting in the sums over $m$ and $n$ in the above.

The final stage is to apply Lemma 6.9 to each of the $r_{<}$integrals, where, by the choice $m=p=2$ for the preceding lemmas, the coefficient $m$ in Lemma 6.9 is either 2 or $4 / 3$, both of which are greater than 1 .

Hence all powers of $\ell$ in terms of $k$ and $k^{\prime}$ cancel. The calculation of the powers of $\ell$ and $r_{>}$is trivial by following the application of the above lemmas.

We will later choose $g\left(r_{<}, r_{>}\right)$to be $A_{j}\left(r_{<}, r_{>}\right)$or $\frac{\partial}{\partial r_{\gamma}} A_{j}\left(r_{<}, r_{>}\right)$. It remains to estimate the decay rate of the norm of the remainder and its derivatives in (5.7) and (5.8).

6.6. Remainder decay rates with assumptions. We now wish to determine the rate of decay of the remainder terms $R_{2 J-1, \ell}\left(r_{<}, r_{>}\right)$and $\frac{\partial}{\partial r_{\gamma}} R_{2 J, \ell}\left(r_{<}, r_{>}\right)$in Theorems 4.2 and 4.3. In particular, from (5.7) and (5.8), we see that we are interested in the integrals

$$
\iint\left|R_{2 J-1, \ell}\left(r_{<}, r_{>}\right)\right|^{2} r_{<}^{2} r_{>}^{2} d r_{<} d r_{>} \text {and } \iint\left|\frac{\partial}{\partial r_{\gamma}} R_{2 J, \ell}\left(r_{<}, r_{>}\right)\right|^{2} r_{<}^{2} r_{>}^{2} d r_{<} d r_{>} .
$$

For much of this section we follow the method used by Hill, starting with the definition of the differential operators $\mathcal{L}_{j}$ ([11, p. 1181]), which are chosen so that $\mathcal{L}_{2 n}$ is equivalent to $n$ applications of the angular Laplacian. We have the recursive definitions

$$
\mathcal{L}_{1}:=\frac{\partial}{\partial \theta}, \quad \mathcal{L}_{2 n}:=\left(\frac{\partial}{\partial \theta}+\frac{\cos \theta}{\sin \theta}\right) \mathcal{L}_{2 n-1}, \quad \mathcal{L}_{2 n+1}:=\frac{\partial}{\partial \theta} \mathcal{L}_{2 n}
$$

and it is clear that $\mathcal{L}_{2 n} \Phi_{\ell}(\theta)=[-\ell(\ell+1)]^{n} \Phi_{\ell}(\theta)$. Consider now an integral of the 
form

$$
I:=\int_{0}^{\pi} f(\theta) \Phi_{\ell}(\theta) \sin \theta d \theta
$$

(where $f(\theta)$ will be $R_{2 J-1}\left(r_{<}, r_{>}, r_{12}\right)$ or $\frac{\partial}{\partial r_{\gamma}} R_{2 J}\left(r_{<}, r_{>}, r_{12}\right)$ ), for which we have

$$
[-\ell(\ell+1)]^{J} I=\int_{0}^{\pi} f(\theta)\left(\mathcal{L}_{2 J} \Phi_{\ell}(\theta)\right) \sin \theta d \theta .
$$

We now wish to rewrite this integral so that the operators $\mathcal{L}_{j}$ apply to $f(\theta)$ rather than to $\Phi_{\ell}(\theta)$.

LEMMA 6.11. For a general $2 J$-times differentiable function $f(\theta)$ and operators $\mathcal{L}_{j}$ defined in (6.44) we have

$$
\begin{aligned}
\int_{0}^{\pi} f(\theta)\left(\mathcal{L}_{2 J} \Phi_{\ell}(\theta)\right) \sin \theta d \theta= & {\left[\sin \theta \sum_{j=0}^{2 J-1}(-1)^{j}\left(\mathcal{L}_{j} f(\theta)\right)\left(\mathcal{L}_{2 J-1-j} \Phi_{\ell}(\theta)\right)\right]_{0}^{\pi} } \\
& +\int_{0}^{\pi}\left(\mathcal{L}_{2 J} f(\theta)\right) \Phi_{\ell}(\theta) \sin \theta d \theta
\end{aligned}
$$

Proof. This follows trivially by induction on $J$, using integration by parts and (6.44).

We now note that as long as $\left(\mathcal{L}_{j} f(\theta)\right)\left(\mathcal{L}_{2 J-1-j} \Phi_{\ell}(\theta)\right)$ is bounded for all $j=$ $0, \ldots, 2 J-1$, then the boundary terms in Lemma 6.11 are zero. We now have the following lemmas.

LEMMA 6.12. Suppose that

$$
\left|\left(\mathcal{L}_{j} R_{2 J-1}\left(r_{<}, r_{>}, r_{12}\right)\right)\left(\mathcal{L}_{2 J-1-j} \Phi_{\ell}(\theta)\right)\right|<\infty
$$

for $j=0, \ldots, 2 J-1$, then

$$
\sum_{\ell=0}^{\infty} \ell^{4 J}\left|R_{2 J-1, \ell}\left(r_{<}, r_{>}\right)\right|^{2} \leq \int_{r_{>}-r_{<}}^{r_{>}+r_{<}}\left|\mathcal{L}_{2 J} R_{2 J-1}\left(r_{<}, r_{>}, r_{12}\right)\right|^{2} \frac{r_{12}}{r_{<} r_{>}} d r_{12} .
$$

Further, if the integral on the right-hand side is finite, then

$$
\lim _{\ell \rightarrow \infty} \ell^{2 J} R_{2 J-1, \ell}\left(r_{<}, r_{>}\right)=0
$$

and if, in addition,

$$
\int_{0}^{\infty} \int_{0}^{r_{>}} \int_{r_{>}-r_{<}}^{r_{>}+r_{<}}\left|\mathcal{L}_{2 J} R_{2 J-1}\left(r_{<}, r_{>}, r_{12}\right)\right|^{2} \frac{r_{12}}{r_{<} r_{>}} d r_{12} r_{<}^{2} r_{>}^{2} d r_{<} d r_{>}<\infty,
$$

then

$$
\lim _{\ell \rightarrow \infty} \ell^{4 J} \int_{0}^{\infty} \int_{0}^{r>}\left|R_{2 J-1, \ell}\left(r_{<}, r_{>}\right)\right|^{2} r_{<}^{2} r_{>}^{2} d r_{<} d r_{>}=0
$$

Proof. Using (6.45) with $f(\theta)=R_{2 J-1}\left(r_{<}, r_{>}, r_{12}\right)$ (i.e., $\left.I=R_{2 J-1, \ell}\left(r_{<}, r_{>}\right)\right)$, along with (6.46), Lemma 6.11 shows that

$$
[-\ell(\ell+1)]^{J} R_{2 J-1, \ell}\left(r_{<}, r_{>}\right)=\int_{0}^{\pi}\left(\mathcal{L}_{2 J} R_{2 J-1}\left(r_{<}, r_{>}, r_{12}\right)\right) \Phi_{\ell}(\theta) \sin \theta d \theta .
$$

Copyright (C) by SIAM. Unauthorized reproduction of this article is prohibited. 
Since $\mathcal{L}_{2 J} R_{2 J-1}\left(r_{<}, r_{>}, r_{12}\right)$ is a function of $r_{<}, r_{>}$and $\theta$, it may be expanded as

$$
\mathcal{L}_{2 J} R_{2 J-1}\left(r_{<}, r_{>}, r_{12}\right)=\sum_{i=0}^{\infty} \alpha_{i}\left(r_{<}, r_{>}\right) \Phi_{i}(\theta) .
$$

Hence, by (6.49), $[\ell(\ell+1)]^{2 J}\left|R_{2 J-1, \ell}\left(r_{<}, r_{>}\right)\right|^{2}=\left|\alpha_{\ell}\left(r_{<}, r_{>}\right)\right|^{2}$. Summing over $\ell$ gives

$$
\sum_{\ell=0}^{\infty}[\ell(\ell+1)]^{2 J}\left|R_{2 J-1, \ell}\left(r_{<}, r_{>}\right)\right|^{2}=\sum_{\ell=0}^{\infty}\left|\alpha_{\ell}\left(r_{<}, r_{>}\right)\right|^{2} .
$$

By definition, and since the $\Phi_{\ell}(\theta)$ are real,

$$
\left|\mathcal{L}_{2 J} R_{2 J-1}\left(r_{<}, r_{>}, r_{12}\right)\right|^{2}=\sum_{i, j=1}^{\infty} \alpha_{i}\left(r_{<}, r_{>}\right) \alpha_{j}^{*}\left(r_{<}, r_{>}\right) \Phi_{i}(\theta) \Phi_{j}(\theta)
$$

Using the orthonormality of the $\Phi_{i}(\theta)$ and the dominated convergence theorem, we have that, if the integral of the left-hand side is bounded,

$$
\int_{0}^{\pi}\left|\mathcal{L}_{2 J} R_{2 J-1}\left(r_{<}, r_{>}, r_{12}\right)\right|^{2} \sin \theta d \theta=\sum_{\ell=0}^{\infty}\left|\alpha_{\ell}\left(r_{<}, r_{>}\right)\right|^{2} .
$$

Since $\ell^{4 J} \leq[\ell(\ell+1)]^{2 J},(6.47)$ follows from (6.50) and (6.51), along with the identity

$$
\int_{0}^{\pi} f \sin \theta d \theta=\int_{r_{>}-r_{<}}^{r_{<}+r_{>}} f \frac{\partial \theta}{\partial r_{12}} \sin \theta d r_{12}=\int_{r_{>}-r_{<}}^{r_{<}+r_{>}} f \frac{r_{12}}{r_{<} r_{>}} d r_{12} .
$$

The second part follows trivially as, if the sum is bounded, the summand must tend to zero and hence $\lim _{\ell \rightarrow \infty} \ell^{2} R_{2 J-1, \ell}\left(r_{<}, r_{>}\right)=0$.

For the final part, let $f_{n}=\sum_{\ell=0}^{n} \ell^{4}\left|R_{2 J-1, \ell}\left(r_{<}, r_{>}\right)\right|^{2}$ then it is trivial that $f_{n} \rightarrow$ $f:=\sum_{\ell=0}^{\infty} \ell^{4}\left|R_{2 J-1, \ell}\left(r_{<}, r_{>}\right)\right|^{2}$ pointwise as $n \rightarrow \infty$. Further, by the positivity of the summand,

$$
\left|f_{n}\right| \leq f \leq \int_{0}^{\pi}\left|\mathcal{L}_{2 J} R_{2 J-1}\left(r_{<}, r_{>}, r_{12}\right)\right|^{2} \sin \theta d \theta=: g .
$$

By assumption $\int_{0}^{\infty} \int_{0}^{r} g r_{<}^{2} r_{>}^{2} d r_{<} d r_{>}<\infty$ and, hence, by the dominated convergence theorem, we may exchange the order of summation and integration in

$$
\int_{0}^{\infty} \int_{0}^{r} \sum_{\ell=0}^{\infty} \ell^{4 J}\left|R_{2 J-1, \ell}\left(r_{<}, r_{>}\right)\right|^{2} r_{<}^{2} r_{>}^{2} d r_{<} d r_{>}<\infty
$$

which gives the final part of the result.

LEMMA 6.13. Suppose that

$$
\left|\left(\mathcal{L}_{j} R_{2 J}\left(r_{<}, r_{>}, r_{12}\right)\right)\left(\mathcal{L}_{2 J-j} \Phi_{\ell}(\theta)\right)\right|<\infty
$$

for $j=0, \ldots, 2 J-1$, that $\frac{\partial}{\partial r_{\gamma}} R_{2 J, \ell}\left(r_{<}, r_{>}\right)$and $\frac{\partial}{\partial r_{\gamma}}\left(\mathcal{L}_{2 J} R_{2 J}\left(r_{<}, r_{>}, r_{12}\right)\right)$ exist for $r_{\gamma} \in\left\{r_{<}, r_{>}\right\}$, and that $\frac{\partial}{\partial r_{\gamma}}\left(\mathcal{L}_{2 J} R_{2 J}\left(r_{<}, r_{>}, r_{12}\right)\right)$ is a continuous function of $r_{\gamma}$.

Copyright $@$ by SIAM. Unauthorized reproduction of this article is prohibited. 
Then

$$
\sum_{\ell=0}^{\infty} \ell^{4 J}\left|\frac{\partial}{\partial r_{\gamma}} R_{2 J, \ell}\left(r_{<}, r_{>}\right)\right|^{2} \leq \int_{r_{>}-r_{<}}^{r_{>}+r_{<}}\left|\frac{\partial}{\partial r_{\gamma}}\left(\mathcal{L}_{2 J} R_{2 J}\left(r_{<}, r_{>}, r_{12}\right)\right)\right|^{2} \frac{r_{12}}{r_{<} r_{>}} d r_{12} .
$$

Further, if the integral on the right-hand side is finite, then

$$
\lim _{\ell \rightarrow \infty} \ell^{2 J} \frac{\partial}{\partial r_{\gamma}} R_{2 J, \ell}\left(r_{<}, r_{>}\right)=0
$$

and if, in addition,

$$
\int_{0}^{\infty} \int_{0}^{r_{>}} \int_{r_{>}-r_{<}}^{r_{>}+r_{<}}\left|\frac{\partial}{\partial r_{\gamma}}\left(\mathcal{L}_{2 J} R_{2 J}\left(r_{<}, r_{>}, r_{12}\right)\right)\right|^{2} \frac{r_{12}}{r_{<} r_{>}} d r_{12} r_{<}^{2} r_{>}^{2} d r_{<} d r_{>}<\infty
$$

then

$$
\lim _{\ell \rightarrow \infty} \ell^{4 J} \int_{0}^{\infty} \int_{0}^{r_{>}}\left|\frac{\partial}{\partial r_{\gamma}} R_{2 J, \ell}\left(r_{<}, r_{>}\right)\right|^{2} r_{<}^{2} r_{>}^{2} d r_{<} d r_{>}=0
$$

Proof. As in the proof of Lemma 6.12 we find

$$
[-\ell(\ell+1)]^{J} R_{2 J, \ell}\left(r_{<}, r_{>}\right)=\int_{0}^{\pi}\left(\mathcal{L}_{2 J} R_{2 J}\left(r_{<}, r_{>}, r_{12}\right)\right) \Phi_{\ell}(\theta) \sin \theta d \theta
$$

Differentiating both sides with respect to $r_{\gamma}$ and, by the continuity of $\mathcal{L}_{2 J} R_{2 J}\left(r_{<}, r_{>}, r_{12}\right)$ and $\frac{\partial}{\partial r_{\gamma}}\left(\mathcal{L}_{2 J} R_{2 J}\left(r_{<}, r_{>}, r_{12}\right)\right)$, using Leibniz's rule [1] to take the derivative inside the integral gives

$$
[-\ell(\ell+1)]^{J} \frac{\partial}{\partial r_{\gamma}} R_{2 J, \ell}\left(r_{<}, r_{>}\right)=\int_{0}^{\pi}\left[\frac{\partial}{\partial r_{\gamma}}\left(\mathcal{L}_{2 J} R_{2 J}\left(r_{<}, r_{>}, r_{12}\right)\right)\right] \Phi_{\ell}(\theta) \sin \theta d \theta
$$

The rest of the proof is analogous to that of Lemma 6.12.

6.7. Validity of assumptions. It is clear from the statements of Lemmas 6.12 and 6.13 that we wish to show that (6.46) holds and that the integrals in (6.47) and (6.52) are finite. The other necessary assumptions will turn out to be more technical assumptions on the wavefunction.

For convenience we follow Hill in defining the more general function

$$
h_{m}^{n}\left(r_{<}, r_{>}, r_{12}\right):=\int_{r_{>}-r_{<}}^{r_{12}} \frac{\left(r_{12}-t\right)^{m}}{m !} \frac{\partial^{n}}{\partial r_{12}^{n}} \psi\left(r_{<}, r_{>}, t\right) d t, m \geq 0
$$

where, in particular, $R_{J}\left(r_{<}, r_{>}, r_{12}\right)=h_{J}^{J+1}\left(r_{<}, r_{>}, r_{12}\right)$. Using Leibniz's rule,

$$
\frac{\partial}{\partial r_{12}} h_{m}^{n}\left(r_{<}, r_{>}, r_{12}\right)=h_{m-1}^{n}\left(r_{<}, r_{>}, r_{12}\right)
$$

and so we also define

$$
h_{-1}^{n}\left(r_{<}, r_{>}, r_{12}\right):=\frac{\partial^{n}}{\partial r_{12}^{n}} \psi\left(r_{<}, r_{>}, r_{12}\right)
$$

Copyright (C) by SIAM. Unauthorized reproduction of this article is prohibited. 
The aim of the next step is to write the operator $\mathcal{L}_{j}$, which is in terms of $\frac{\partial}{\partial \theta}$, in terms of $\frac{\partial}{\partial r_{12}}$, allowing us to make use of (6.55). The following lemma follows Hill's Appendix C.

LEMma 6.14. The operator $\mathcal{L}_{j}$, as defined recursively in (6.44), may be rewritten

$$
\mathcal{L}_{j}=\sum_{k=1}^{j} u_{k}^{j}\left(r_{<}, r_{>}, r_{12}\right) \frac{\partial^{k}}{\partial r_{12}^{k}}
$$

where the $u_{k}^{j}\left(r_{<}, r_{>}, r_{12}\right)$ are given recursively by

$$
\begin{aligned}
u_{k}^{j}\left(r_{<}, r_{>}, r_{12}\right) & :=0, \text { if } k<1 \text { or } k>j, \\
u_{1}^{1}\left(r_{<}, r_{>}, r_{12}\right) & :=\frac{\partial r_{12}}{\partial \theta} \\
u_{k}^{2 j}\left(r_{<}, r_{>}, r_{12}\right) & :=\left(\frac{\partial}{\partial \theta}+\frac{\cos \theta}{\sin \theta}\right) u_{k}^{2 j-1}\left(r_{<}, r_{>}, r_{12}\right)+\frac{\partial r_{12}}{\partial \theta} u_{k-1}^{2 j-1}\left(r_{<}, r_{>}, r_{12}\right), \\
u_{k}^{2 j+1}\left(r_{<}, r_{>}, r_{12}\right) & :=\frac{\partial}{\partial \theta} u_{k}^{2 j}\left(r_{<}, r_{>}, r_{12}\right)+\frac{\partial r_{12}}{\partial \theta} u_{k-1}^{2 j}\left(r_{<}, r_{>}, r_{12}\right) .
\end{aligned}
$$

Proof. The proof follows trivially by induction and the definitions (6.44).

It follows directly from this lemma that we may write

$$
\mathcal{L}_{j} h_{m}^{n}\left(r_{<}, r_{>}, r_{12}\right)=\sum_{k=1}^{j} h_{m-k}^{n}\left(r_{<}, r_{>}, r_{12}\right) u_{k}^{j}\left(r_{<}, r_{>}, r_{12}\right),
$$

and hence we are interested in the boundedness of the $u_{k}^{j}\left(r_{<}, r_{>}, r_{12}\right)$. At present we have an expression for $u_{k}^{j}\left(r_{<}, r_{>}, r_{12}\right)$ with terms in both $r_{12}$ and $\theta$, which can be related in terms of $r_{<}$and $r_{>}$. Our next step is therefore to write $u_{k}^{j}\left(r_{<}, r_{>}, r_{12}\right)$ in only $r_{<}, r_{>}$and $r_{12}$; this again follows Appendix $\mathrm{C}$ of [11].

Lemma 6.15. The $u_{k}^{j}\left(r_{<}, r_{>}, r_{12}\right)$ defined in Lemma 6.14 are given by

$$
u_{k}^{j}\left(r_{<}, r_{>}, r_{12}\right)=\sum_{i=0}^{j-k} \frac{\left(r_{<} r_{>}\right)^{k+i}}{r_{12}^{k+2 i}} v_{k, i}^{j}(\theta)
$$

where the $v_{k, i}^{j}(\theta)$ are given recursively by

$$
\begin{aligned}
v_{k, i}^{j}(\theta) & =0, \text { if } i<0 \text { or } i>j-k \\
v_{1,0}^{1}(\theta) & =\sin \theta \\
v_{k, i}^{2 j}(\theta) & =\left(\frac{\partial}{\partial \theta}+\frac{\cos \theta}{\sin \theta}\right) v_{k, i}^{2 j-1}(\theta)+\sin \theta\left[v_{k-1, i}^{2 j-1}(\theta)-(k+2 i-2) v_{k, i-1}^{2 j-1}(\theta)\right], \\
v_{k, i}^{2 j+1}(\theta) & =\frac{\partial}{\partial \theta} v_{k, i}^{2 j}(\theta)+\sin \theta\left[v_{k-1, i}^{2 j}(\theta)-(k+2 i-2) v_{k, i}^{2 j-1}(\theta)\right] .
\end{aligned}
$$

Proof. The result follows trivially by induction and $\frac{\partial r_{12}}{\partial \theta}=r_{<} r_{>} \sin \theta / r_{12}$.

The final stage necessary to prove the boundedness of $\mathcal{L}_{j} h_{m}^{n}\left(r_{<}, r_{>}, r_{12}\right)$ is to derive a more explicit form for $v_{k, i}^{j}(\theta)$. For this stage of the proof we deviate somewhat from [11] and derive the minimal degree of the $v_{k, i}^{j}(\theta)$ as polynomials in $\sin \theta$ more directly. In particular we wish to show the following.

Copyright $@$ by SIAM. Unauthorized reproduction of this article is prohibited. 
LEMMA 6.16. Let

$$
\begin{aligned}
\mu_{k, i}^{2 j} & :=\max \{0,2(k+i-j)\}, \\
\mu_{k, i}^{2 j+1} & :=\max \{1,2(k+i-j)-1\},
\end{aligned}
$$

and then for $1 \leq k \leq j$ and $0 \leq i \leq j-k, v_{k, i}^{j}(\theta)$ are of the form

$$
v_{k, i}^{j}(\theta)= \begin{cases}\frac{1}{2\left(k+i-\mu_{k, i}^{j}\right)} a_{k, i, n}^{j}(\sin \theta)^{2 n+\mu_{k, i}^{j}} & \text { if } j+k+i \text { even } \\ \sum_{n=0}^{\frac{1}{2}\left(k+i-1-\mu_{k, i}^{j}\right)} a_{k, i, n}^{j}(\sin \theta)^{2 n+\mu_{k, i}^{j} \cos \theta} & \text { if } j+k+i \text { odd },\end{cases}
$$

where $a_{k, i, n}^{j} \in \mathbb{R}$, and else $v_{k, i}^{j}(\theta)=0$.

Proof. The cases where $v_{k, i}^{j}(\theta)=0$ follow immediately from their definition in Lemma 6.15. It is also worth noting that the upper limits of the sums are integers since $\mu_{k, i}^{j}$ is of the same parity as $j$.

For the other cases, proceeding by induction, for $j=1$ we need only consider the case $v_{1,0}^{1}(\theta)=\sin \theta$ where $\mu_{1,0}^{1}=1, j+k+i=2, k+i-\mu_{1,0}^{1}=0$, and so the result holds with $a_{1,0,0}^{1}=1$.

Now, assuming that the result holds up to $2 j-1$, we see from (6.62) that

$$
v_{k, i}^{2 j}(\theta)=\left(\frac{\partial}{\partial \theta}+\frac{\cos \theta}{\sin \theta}\right) v_{k, i}^{2 j-1}(\theta)+\sin \theta\left[v_{k-1, i}^{2 j-1}(\theta)-(k+2 i-2) v_{k, i-1}^{2 j-1}(\theta)\right]
$$

so to construct $v_{k, i}^{2 j}(\theta)$ we require $v_{k, i}^{2 j-1}(\theta), v_{k-1, i}^{2 j-1}(\theta)$, and $v_{k, i-1}^{2 j-1}(\theta)$. It is useful at this stage to compare

$$
\begin{aligned}
\mu_{k, i}^{2 j-1} & =\max \{1,2(k+i-j)+1\}, \\
\mu_{k-1, i}^{2 j-1}=\mu_{k, i-1}^{2 j-1} & =\max \{1,2(k+i-j)-1\}, \text { and } \\
\mu_{k, i}^{2 j} & =\max \{0,2(k+i-j)\}=\mu_{k, i}^{2 j-1}-1 .
\end{aligned}
$$

We now consider two cases, firstly, if $\mu_{k, i}^{2 j-1}=1$, then we have

$$
\mu_{k, i}^{2 j-1}=\mu_{k-1, i}^{2 j-1}=\mu_{k, i-1}^{2 j-1}=1, \quad \mu_{k, i}^{2 j}=0 .
$$

Secondly, if $\mu_{k, i}^{2 j-1}>1$, then, by its oddness, $\mu_{k, i}^{2 j-1} \geq 3$ and hence $2(k+i-j)-1 \geq 1$, giving

$$
\mu_{k, i}^{2 j-1}=\mu_{k, i}^{2 j}+1, \quad \mu_{k-1, i}^{2 j-1}=\mu_{k, i-1}^{2 j-1}=\mu_{k, i}^{2 j-1}-2=\mu_{k, i}^{2 j}-1
$$

Recalling that we are constructing $v_{k, i}^{2 j}(\theta)$ from $v_{k, i}^{2 j-1}(\theta)$, there are two cases to consider depending on whether $2 j-1+k+i$ is odd or even. Suppose first that $2 j-1+k+i$ is odd (so $2 j-k+i-2$ is even). Using (6.64) for $v_{k, i}^{2 j-1}(\theta)$, the first term of (6.65) is, up to a constant,

$$
\begin{aligned}
& \left(\frac{\partial}{\partial \theta}+\frac{\cos \theta}{\sin \theta}\right)(\sin \theta)^{2 n+\mu_{k, i}^{2 j-1}} \cos \theta \\
& \quad=\left(2 n+\mu_{k, i}^{2 j-1}+1\right)(\sin \theta)^{2 n+\mu_{k, i}^{2 j-1}-1}-\left(2 n+\mu_{k, i}^{2 j-1}+2\right)(\sin \theta)^{2 n+\mu_{k, i}^{2 j-1}+1}
\end{aligned}
$$

Copyright (C) by SIAM. Unauthorized reproduction of this article is prohibited. 
Using that $\mu_{k-1, i}^{2 j-1}=\mu_{k, i-1}^{2 j-1}$ and (6.64) for $v_{k-1, i}^{2 j-1}(\theta)$ and $v_{k, i-1}^{2 j-1}(\theta)$, the second term of (6.65) contributes, up to a constant, $(\sin \theta)^{2 n+\mu_{k-1, i}^{2 j-1}+1}$. These two results lead to an expression for $v_{k, i}^{2 j}(\theta)$ of the form

$$
\begin{aligned}
v_{k, i}^{2 j}(\theta)= & \sum_{n=0}^{\frac{1}{2}\left(k+i-1-\mu_{k, i}^{2 j-1}\right)} \alpha_{n}(\sin \theta)^{2 n+\mu_{k, i}^{2 j-1}-1}+\beta_{n}(\sin \theta)^{2 n+\mu_{k, i}^{2 j-1}+1} \\
& +\sum_{n=0}^{\frac{1}{2}\left(k+i-1-\mu_{k-1, i}^{2 j-1}\right)} \gamma_{n}(\sin \theta)^{2 n+\mu_{k-1, i}^{2 j-1}+1} .
\end{aligned}
$$

Considering separately the cases $\mu_{k, i}^{2 j-1}=1$ (using (6.66), and $\left.\mu_{k, i}^{2 j}=0\right)$ and $\mu_{k, i}^{2 j-1}>1$ (using (6.67)), we see that, for some $a_{k, i, n}^{j}$, this becomes

$$
v_{k, i}^{2 j}(\theta)=\sum_{n=0}^{\frac{1}{2}\left(k+i-\mu_{k, i}^{2 j}\right)} a_{k, i, n}^{2 j}(\sin \theta)^{2 n+\mu_{k, i}^{2 j}} .
$$

This proves the case for $2 j-1 \rightarrow 2 j$ with $2 j-1+k+i$ odd. The other results follow with almost identical proofs and the use of (6.63).

The important point of Lemma 6.16 is that it shows that the lowest power of $\sin \theta$ in $v_{k, i}^{j}(\theta)$ is given by $\mu_{k, i}^{j}$. Writing $\mu_{k, i}^{2 j}=\max \{0,2(k+i)-2 j\}$ and $\mu_{k, i}^{2 j+1}=$ $\max \{1,2(k+i)-(2 j+1)\}$ it is clear that, for some positive constants $C_{k, i}^{j}$,

$$
v_{k, i}^{j}(\theta) \leq \begin{cases}C_{k, i}^{j} & \text { if } 2 k+2 i-j \leq 0 \\ C_{k, i}^{j}(\sin \theta)^{2 k+2 i-j} & \text { if } 2 k+2 i-j>0\end{cases}
$$

We wish to use this result to prove a bound on $u_{k}^{j}\left(r_{<}, r_{>}, r_{12}\right)$.

Lemma 6.17. For $u_{k}^{j}\left(r_{<}, r_{>}, r_{12}\right)$ as defined in Lemma 6.14 and positive constants $C_{k, i}^{j}$ as defined in (6.68), and defining $C_{k}^{j}=\sum_{i=0}^{j-k} C_{k, i}^{j}$,

$$
\left|u_{k}^{j}\left(r_{<}, r_{>}, r_{12}\right)\right| \leq C_{k}^{j}\left(\frac{r_{<} r_{>}}{r_{12}}\right)^{1 / 2}\left(r_{<}+r_{>}\right)^{j-1} r_{12}^{k-j+1 / 2} .
$$

Proof. Consider first the case $2 k+2 i-j \leq 0$. Inserting the bound in (6.68) into (6.61), and using the trivial inequality $r_{12} \leq r_{>}+r_{<}$, we see that

$$
\begin{aligned}
\left|\frac{\left(r_{<} r_{>}\right)^{k+i}}{r_{12}^{k+2 i}} v_{k, i}^{j}(\theta)\right| & \leq C_{k, i}^{j} \frac{\left(r_{<} r_{>}\right)^{k+i}}{r_{12}^{k+2 i}}\left(\frac{r_{<}+r_{>}}{r_{12}}\right)^{j-2 k-2 i} \\
& =C_{k, i}^{j}\left(r_{<} r_{>}\right)^{k+i}\left(r_{<}+r_{>}\right)^{j-2 k-2 i} r_{12}^{k-j} .
\end{aligned}
$$

By the arithmetic-geometric inequality

$$
\left(r_{<} r_{>}\right)^{1 / 2} \leq r_{<}+r_{>}
$$

we have

$$
\left|\frac{\left(r_{<} r_{>}\right)^{k+i}}{r_{12}^{k+2 i}} v_{k, i}^{j}(\theta)\right| \leq C_{k, i}^{j}\left(\frac{r_{<} r_{>}}{r_{12}}\right)^{1 / 2}\left(r_{<}+r_{>}\right)^{j-1} r_{12}^{k-j+1 / 2},
$$

where $\left(r_{<} r_{>} / r_{12}\right)^{1 / 2}$ is kept so as to cancel with the analogous term in (6.47).

Copyright (c) by SIAM. Unauthorized reproduction of this article is prohibited. 
For the case where $2 k+2 i-j>0$ we use the inequality

$$
\sin \theta \leq r_{12}\left(r_{<} r_{>}\right)^{-1 / 2}
$$

(which is easily derived from the sine rule) along with (6.70) to give

$$
\begin{aligned}
\left|\frac{\left(r_{<} r_{>}\right)^{k+i}}{r_{12}^{k+2 i}} v_{k, i}^{j}(\theta)\right| & \leq C_{k, i}^{j} \frac{\left(r_{<} r_{>}\right)^{k+i}}{r_{12}^{k+2 i}}(\sin \theta)^{2 k+2 i-j} \\
& \leq C_{k, i}^{j} \frac{\left(r_{<} r_{>}\right)^{k+i}}{r_{12}^{k+2 i}} \frac{r_{12}^{2 k+2 i-j}}{\left(r_{<} r_{>}\right)^{k+i-j / 2}}=C_{k, i}^{j}\left(r_{<} r_{>}\right)^{j / 2} r_{12}^{k-j} . \\
& \leq C_{k, i}^{j}\left(\frac{r_{<} r_{>}}{r_{12}}\right)^{1 / 2}\left(r_{<}+r_{>}\right)^{j-1} r_{12}^{k-j+1 / 2},
\end{aligned}
$$

which is identical to (6.71). Noting that this bound is independent of $i$ and using (6.61) we have

$$
\left|u_{k}^{j}\left(r_{<}, r_{>}, r_{12}\right)\right| \leq C_{k}^{j}\left(\frac{r_{<} r_{>}}{r_{12}}\right)^{1 / 2}\left(r_{<}+r_{>}\right)^{j-1} r_{12}^{k-j+1 / 2},
$$

where $C_{k}^{j}=\sum_{i=0}^{j-k} C_{k, i}^{j}$, proving the result.

We now prove an analogous result for the derivatives of $u_{k}^{j}\left(r_{<}, r_{>}, r_{12}\right)$.

LEMMA 6.18. For $\frac{\partial}{\partial r_{\gamma}} u_{k}^{j}\left(r_{<}, r_{>}, r_{12}\right)$ with $u_{k}^{j}\left(r_{<}, r_{>}, r_{12}\right)$, as defined in Lemma 6.14 and $r_{\gamma} \in\left\{r_{<}, r_{>}\right\}$, there exist positive constants $\tilde{C}_{k, \gamma}^{j}$ such that

$$
\left|\frac{\partial}{\partial r_{\gamma}} u_{k}^{j}\left(r_{<}, r_{>}, r_{12}\right)\right| \leq \tilde{C}_{k, \gamma}^{j}\left(\frac{r_{<} r_{>}}{r_{12}}\right)^{1 / 2}\left(r_{<}+r_{>}\right)^{j-1} r_{12}^{k-j-\frac{1}{2}} .
$$

Proof. We start with the case $r_{\gamma}=r_{<}$. From (6.61) we see that

$$
\begin{aligned}
\frac{\partial}{\partial r_{<}} u_{k}^{j}\left(r_{<}, r_{>}, r_{12}\right) & =\sum_{i=0}^{j-k}\left[\frac{\partial r_{12}}{\partial r_{<}}(-k-2 i) \frac{\left(r_{<} r_{>}\right)^{k+i}}{r_{12}^{k+2 i+1}}+(k+i) r_{>} \frac{\left(r_{<} r_{>}\right)^{k+i-1}}{r_{12}^{k+2 i}}\right] v_{k, i}^{j}(\theta) \\
& =\sum_{i=0}^{j-k}\left[(-k-2 i) \frac{\partial r_{12}}{\partial r_{<}} r_{<} r_{>}+(k+i) r_{12} r_{>}\right] \frac{\left(r_{<} r_{>}\right)^{k+i-1}}{r_{12}^{k+2 i+1}} v_{k, i}^{j}(\theta) .
\end{aligned}
$$

Now note that $\left|\partial r_{12} / \partial r_{<}\right| \leq 1$, which follows trivially from the geometric interpretation of $r_{12}$, the triangle inequality and the definition of the derivative (or from considering the algebraic form of $\left.\left(\partial r_{12} / \partial r_{<}\right)^{2}\right)$.

Using the inequalities $r_{>} \leq r_{<}+r_{>}, r_{12} \leq r_{<}+r_{>}$, and $\left(r_{<} r_{>}\right)^{1 / 2} \leq r_{>}+r_{<}$, we therefore have that

$$
\left|\frac{\partial}{\partial r_{<}} u_{k}^{j}\left(r_{<}, r_{>}, r_{12}\right)\right| \leq \sum_{i=0}^{j-k} \tilde{C}_{k, i}^{j}\left|\frac{\left(r_{<} r_{>}\right)^{k+i-1}}{r_{12}^{k+2 i+1}}\left(r_{<}+r_{>}\right)^{2}\right|\left|v_{k, i}^{j}(\theta)\right|
$$

for some positive constants $\tilde{C}_{k, i}^{j}$. The rest of the proof is virtually identical to that of Lemma 6.17. The proof for $r_{\gamma}=r_{>}$is analogous.

These bounds on $u_{k}^{j}\left(r_{<}, r_{>}, r_{12}\right)$ and $\frac{\partial}{\partial r_{\gamma}} u_{k}^{j}\left(r_{<}, r_{>}, r_{12}\right)$ are sufficient to show that the assumptions necessary for Lemma 6.12 hold, namely that.

Copyright (c) by SIAM. Unauthorized reproduction of this article is prohibited. 
Lemma 6.19. For $j=0, \ldots, m$ there exist constants $0<C^{j}<\infty$ such that

$$
\left|\mathcal{L}_{j} h_{m}^{n}\left(r_{<}, r_{>}, r_{12}\right)\right| \leq C^{j} I_{n}^{1 / 2}\left(\frac{r_{<} r_{>}}{r_{12}}\right)^{1 / 2}\left(r_{<}+r_{>}\right)^{j-1} r_{12}^{m+1-j},
$$

where $I_{n}$ are given by (2.12), and $0<C<\infty$ such that

$$
\int_{r_{>}-r_{<}}^{r_{>}+r_{<}}\left|\mathcal{L}_{m+1} h_{m}^{n}\left(r_{<}, r_{>}, r_{12}\right)\right|^{2} \frac{r_{12}}{r_{<} r_{>}} d r_{12} \leq C r_{>}^{2 m+1} I_{n}
$$

Furthermore, there exists $0<\tilde{C}<\infty$ such that

$$
\int_{r_{>}-r_{<}}^{r_{>}+r_{<}}\left|\frac{\partial}{\partial r_{\gamma}}\left(\mathcal{L}_{m} h_{m}^{n}\left(r_{<}, r_{>}, r_{12}\right)\right)\right|^{2} \frac{r_{12}}{r_{<} r_{>}} d r_{12} \leq \tilde{C} r_{>}^{2 m-1} I_{n} .
$$

Proof. We begin by using the identity (6.60) which implies

$$
\left|\mathcal{L}_{j} h_{m}^{n}\left(r_{<}, r_{>}, r_{12}\right)\right| \leq \sum_{k=1}^{j}\left|h_{m-k}^{n}\left(r_{<}, r_{>}, r_{12}\right) u_{k}^{j}\left(r_{<}, r_{>}, r_{12}\right)\right|,
$$

and hence the bound on $u_{k}^{j}\left(r_{<}, r_{>}, r_{12}\right)$ given in (6.69) shows that

$$
\left|\mathcal{L}_{j} h_{m}^{n}\left(r_{<}, r_{>}, r_{12}\right)\right| \leq \sum_{k=1}^{j}\left|h_{m-k}^{n}\left(r_{<}, r_{>}, r_{12}\right)\right|\left(\frac{r_{<} r_{>}}{r_{12}}\right)^{1 / 2}\left(r_{<}+r_{>}\right)^{j-1} r_{12}^{k-j+1 / 2} .
$$

Recalling the definition of $h_{m}^{n}\left(r_{<}, r_{>}, r_{12}\right)$ from (6.54) and using the CauchySchwarz inequality we have, for $m \geq 0$,

$$
\begin{aligned}
\left|h_{m}^{n}\left(r_{<}, r_{>}, r_{12}\right)\right| & \leq\left(\int_{r_{>}-r_{<}}^{r_{12}}\left|\frac{\left(r_{12}-t\right)^{m}}{m !}\right|^{2} d t \int_{r_{>}-r_{<}}^{r_{12}}\left|\frac{\partial^{n}}{\partial r_{12}^{n}} \psi\left(r_{<}, r_{>}, t\right)\right|^{2} d t\right)^{1 / 2} \\
& =\frac{\left(r_{12}-\left(r_{>}-r_{<}\right)\right)^{m+1 / 2}}{m ! \sqrt{2 m+1}}\left(\int_{r_{>}-r_{<}}^{r_{<}+r_{>}}\left|\frac{\partial^{n}}{\partial r_{12}^{n}} \psi\left(r_{<}, r_{>}, t\right)\right|^{2} d t\right)^{1 / 2} \\
& \leq \frac{r_{12}^{m+1 / 2}}{m ! \sqrt{2 m+1}}\left(\int_{r_{>}-r_{<}}^{r_{<}+r_{>}}\left|\frac{\partial^{n}}{\partial r_{12}^{n}} \psi\left(r_{<}, r_{>}, t\right)\right|^{2} d t\right)^{1 / 2} .
\end{aligned}
$$

Hence, for $1 \leq k \leq 2 J-1$,

$$
\begin{aligned}
& \quad\left|h_{m-k}^{n}\left(r_{<}, r_{>}, r_{12}\right) u_{k}^{j}\left(r_{<}, r_{>}, r_{12}\right)\right| \\
& \quad \leq I_{n}^{1 / 2} \frac{C_{k}^{j}}{(m-k) ! \sqrt{2 m-2 k+1}}\left(r_{<} r_{>}\right)^{1 / 2}\left(r_{<}+r_{>}\right)^{j-1} r_{12}^{m-j+1 / 2} .
\end{aligned}
$$

We note that, since $j \leq m$, the power of $r_{12}$ is positive, preventing a singularity at $r_{12}=0$. The first part of the result, the bound on $\mathcal{L}_{j} h_{m}^{n}\left(r_{<}, r_{>}, r_{12}\right)$ for $1 \leq j \leq$ $m$, follows from the expansion (6.75) and the bound (6.77) (which, apart from the constant, is independent of $k$ ). In particular,

$$
C^{j}:=\sum_{k=1}^{j} \frac{C_{k}^{j}}{(m-k) ! \sqrt{2 m-2 k+1}} .
$$

Copyright $@$ by SIAM. Unauthorized reproduction of this article is prohibited. 
The case $j=0$ follows directly from the bound (6.76). This proves the first part of the result.

The above bound does not apply for $k=m+1$ as the Cauchy-Schwarz estimate is not valid. However, since $u_{1}^{1}\left(r_{<}, r_{>}, r_{12}\right)=\frac{\partial r_{12}}{\partial \theta}$ and $u_{j+1}^{j}\left(r_{<}, r_{>}, r_{12}\right)=0$, using (6.58) and (6.59), we have $u_{j}^{j}\left(r_{<}, r_{>}, r_{12}\right)=\left(\frac{\partial r_{12}}{\partial \theta}\right)^{j}$. Hence, using the definition of $h_{-1}^{n}\left(r_{<}, r_{>}, r_{12}\right)$ in (6.56), and $d r_{12} / d \theta=r_{<} r_{>} \sin \theta r_{12}^{-1}$,

$$
\begin{aligned}
\left|h_{-1}^{n}\left(r_{<}, r_{>}, r_{12}\right) u_{m+1}^{m+1}\left(r_{<}, r_{>}, r_{12}\right)\right| & =\left|\frac{\partial^{n}}{\partial r_{12}^{n}} \psi\left(r_{<}, r_{>}, t\right)\right|\left(\frac{\partial r_{12}}{\partial \theta}\right)^{m+1} \\
& \leq\left|\frac{\partial^{n}}{\partial r_{12}^{n}} \psi\left(r_{<}, r_{>}, t\right)\right|\left(r_{<} r_{>}\right)^{(2 m+1) / 4}\left(\frac{r_{<} r_{>}}{r_{12}}\right)^{1 / 2}
\end{aligned}
$$

where the inequality uses $(6.72)$ and $|\sin \theta| \leq 1$.

To obtain the bound on the integral we use the identity

$$
\mathcal{L}_{m+1} h_{m}^{n}\left(r_{<}, r_{>}, r_{12}\right)=\sum_{k=1}^{m+1} h_{m-k}^{n}\left(r_{<}, r_{>}, r_{12}\right) u_{k}^{m+1}\left(r_{<}, r_{>}, r_{12}\right)
$$

and the bounds (6.77) and (6.79) to obtain

$$
\begin{aligned}
\left|\mathcal{L}_{m+1} h_{m}^{n}\left(r_{<}, r_{>}, r_{12}\right)\right| \leq \sum_{k=1}^{m} & I_{n}^{1 / 2} \frac{C_{k}^{m+1}}{(m-k) ! \sqrt{2 m-2 k+1}}\left(\frac{r_{<} r_{>}}{r_{12}}\right)^{1 / 2}\left(r_{<}+r_{>}\right)^{m} \\
& +\left|\frac{\partial^{n}}{\partial r_{12}^{n}} \psi\left(r_{<}, r_{>}, r_{12}\right)\right|\left(r_{<} r_{>}\right)^{(2 m+1) / 4}\left(\frac{r_{<} r_{>}}{r_{12}}\right)^{1 / 2} \\
\leq & \left(\frac{r_{<} r_{>}}{r_{12}}\right)^{1 / 2}\left[I_{n}^{1 / 2} \tilde{C} r_{>}^{m}+\left|\frac{\partial^{n}}{\partial r_{12}^{n}} \psi\left(r_{<}, r_{>}, r_{12}\right)\right| r_{>}^{(2 m+1) / 2}\right],
\end{aligned}
$$

where the second inequality uses that $r_{<} \leq r_{>}$and the constant $\tilde{C}$ is given by

$$
\tilde{C}:=2^{m} \sum_{k=1}^{m} \frac{C_{k}^{m+1}}{(m-k) ! \sqrt{2 m-2 k+1}} .
$$

Hence we see that

$$
\begin{aligned}
\int_{r_{>}-r_{<}}^{r_{>}+r_{<}}\left|\mathcal{L}_{m+1} h_{m}^{n}\left(r_{<}, r_{>}, r_{12}\right)\right|^{2} \frac{r_{12}}{r_{<} r_{>}} d r_{12} \\
\quad \leq \int_{r_{>}-r_{<}}^{r_{>}+r_{<}}\left[I_{n}^{1 / 2} \tilde{C} r_{>}^{m}+\left|\frac{\partial^{n}}{\partial r_{12}^{n}} \psi\left(r_{<}, r_{>}, r_{12}\right)\right| r_{>}^{(2 m+1) / 2}\right]^{2} d r_{12} \\
\quad \leq I_{n} \tilde{C}^{2} r_{>}^{2 m} \int_{r_{>}-r_{<}}^{r_{>}+r_{<}} d r_{12}+2 I_{n} \tilde{C} r_{>}^{2 m+1 / 2}\left(\int_{r_{>}-r_{<}}^{r_{>}+r_{<}} d r_{12}\right)^{1 / 2}+r_{>}^{2 m+1} I_{n} \\
\quad \leq C I_{n} r_{>}^{2 m+1},
\end{aligned}
$$

where we have used the Cauchy-Schwarz inequality, the definition of $I_{n}$ from (2.12), the fact that $\int_{r_{>}-r_{<}}^{r_{>}+r_{<}} d r_{12}=2 r_{<} \leq 2 r_{>}$, and the definition $C:=2 \tilde{C}^{2}+2 \sqrt{2} \tilde{C}+1=$ $(\sqrt{2} \tilde{C}+1)^{2}$. 
Finally we move on to consider the derivatives of $\mathcal{L}_{m} h_{m}^{n}\left(r_{<}, r_{>}, r_{12}\right)$ and using (6.60) for $j=m$, we see that

$$
\begin{aligned}
\frac{\partial}{\partial r_{\gamma}}\left(\mathcal{L}_{m} h_{m}^{n}\left(r_{<}, r_{>}, r_{12}\right)\right)=\sum_{k=1}^{m} & {\left[h_{m-k}^{n}\left(r_{<}, r_{>}, r_{12}\right) \frac{\partial}{\partial r_{\gamma}} u_{k}^{m}\left(r_{<}, r_{>}, r_{12}\right)\right.} \\
& \left.+u_{k}^{m}\left(r_{<}, r_{>}, r_{12}\right) \frac{\partial}{\partial r_{\gamma}} h_{m-k}^{n}\left(r_{<}, r_{>}, r_{12}\right)\right] .
\end{aligned}
$$

We also have that

$$
\left|\frac{\partial}{\partial r_{\gamma}} h_{m}^{n}\left(r_{<}, r_{>}, r_{12}\right)\right|=\left|\frac{\partial r_{12}}{\partial r_{\gamma}} \frac{\partial}{\partial r_{12}} h_{m}^{n}\left(r_{<}, r_{>}, r_{12}\right)\right| \leq\left|h_{m-1}^{n}\left(r_{<}, r_{>}, r_{12}\right)\right|,
$$

where we have once again used that $\left|\partial r_{12} / \partial r_{\gamma}\right| \leq 1$. The bounds on $\left|h_{m}^{n}\left(r_{<}, r_{>}, r_{12}\right)\right|$, $u_{k}^{j}\left(r_{<}, r_{>}, r_{12}\right)$, and $\frac{\partial}{\partial r_{\gamma}} u_{k}^{j}\left(r_{<}, r_{>}, r_{12}\right)$, from (6.76), and Lemmas 6.17 and 6.18, respectively, give

$$
\left|\frac{\partial}{\partial r_{\gamma}} \mathcal{L}_{m} h_{m}^{n}\left(r_{<}, r_{>}, r_{12}\right)\right| \leq C I_{n}^{1 / 2}\left(r_{<}+r_{>}\right)^{m-1}\left(\frac{r_{<} r_{>}}{r_{12}}\right)^{1 / 2},
$$

for some positive constant $C$. Hence

$$
\begin{gathered}
\int_{r_{>}-r_{<}}^{r_{>}+r_{<}}\left|\frac{\partial}{\partial r_{\gamma}} \mathcal{L}_{m} h_{m}^{n}\left(r_{<}, r_{>}, r_{12}\right)\right|^{2} \frac{r_{12}}{r_{<} r_{>}} d r_{12} \leq C^{2} I_{n}\left(r_{<}+r_{>}\right)^{2 m-2} \int_{r_{>}-r_{<}}^{r_{>}+r_{<}} d r_{12} \\
\leq \tilde{C} r_{>}^{2 m-1} I_{n},
\end{gathered}
$$

giving the result.

6.8. Remainder decay rates. We now have the following result for the remainder terms.

Corollary 6.20. Suppose that

$$
\int_{r_{>}-r_{<}}^{r_{<}+r_{>}}\left|\frac{\partial^{2 J}}{\partial r_{12}^{2 J}} \psi\left(r_{<}, r_{>}, t\right)\right|^{2} d t<\infty,
$$

then $R_{2 J-1, \ell}\left(r_{<}, r_{>}\right)$as given in (2.11) satisfies

$$
\lim _{\ell \rightarrow \infty} \ell^{2 J} R_{2 J-1, \ell}\left(r_{<}, r_{>}\right)=0 .
$$

If, in addition,

$$
\int_{0}^{\infty} \int_{0}^{r_{>}} r_{<}^{4 J-1} \int_{r_{>}-r_{<}}^{r_{<}+r_{>}}\left|\frac{\partial^{2 J}}{\partial r_{12}^{2 J}} \psi\left(r_{<}, r_{>}, t\right)\right|^{2} d t r_{<}^{2} r_{>}^{2} d r_{<} d r_{>}<\infty
$$

then

$$
\lim _{\ell \rightarrow \infty} \ell^{4 J} \int_{0}^{\infty} \int_{0}^{r_{>}}\left|R_{2 J-1, \ell}\left(r_{<}, r_{>}\right)\right|^{2} r_{<}^{2} r_{>}^{2} d r_{<} d r_{>}=0
$$

Proof. We begin by recalling that $R_{2 J-1}\left(r_{<}, r_{>}, r_{12}\right)=h_{2 J-1}^{2 J}\left(r_{<}, r_{>}, r_{12}\right)$ and so, using (6.73) with $m=2 J-1$ and $n=2 J$ shows that (6.80) and (6.81) are

Copyright (c) by SIAM. Unauthorized reproduction of this article is prohibited. 
stronger than the assumption on the right-hand side of (6.47) and the assumption (6.48), respectively.

Furthermore, Lemma 6.19 and the fact that $\mathcal{L}_{j} \Phi_{\ell}(\theta)<\infty$ for all $j$ removes the need for the assumption on the boundedness of $\left|\mathcal{L}_{j} R_{2 J}\left(r_{<}, r_{>}, r_{12}\right)\right|$ in Lemma 6.12.

Hence all assumptions in Lemma 6.12 are satisfied and the result holds.

Corollary 6.21. Suppose that

$$
\int_{r_{>}-r_{<}}^{r_{<}+r_{>}}\left|\frac{\partial^{2 J+1}}{\partial r_{12}^{2 J+1}} \psi\left(r_{<}, r_{>}, t\right)\right|^{2} d t<\infty,
$$

then, for $r_{\gamma} \in\left\{r_{<}, r_{>}\right\}, \frac{\partial}{\partial r_{\gamma}} R_{2 J, \ell}\left(r_{<}, r_{>}\right)$(with $R_{2 J, \ell}\left(r_{<}, r_{>}\right)$from (2.11)) satisfies

$$
\lim _{\ell \rightarrow \infty} \ell^{2 J} \frac{\partial}{\partial r_{\gamma}} R_{2 J, \ell}\left(r_{<}, r_{>}\right)=0 .
$$

If, in addition,

$$
\int_{0}^{\infty} \int_{0}^{r>} r_{<}^{4 J-1} \int_{r_{>}-r_{<}}^{r_{<}+r_{>}}\left|\frac{\partial^{2 J+1}}{\partial r_{12}^{2 J+1}} \psi\left(r_{<}, r_{>}, t\right)\right|^{2} d t r_{<}^{2} r_{>}^{2} d r_{<} d r_{>}<\infty
$$

then

$$
\lim _{\ell \rightarrow \infty} \ell^{4 J} \int_{0}^{\infty} \int_{0}^{r>}\left|\frac{\partial}{\partial r_{\gamma}} R_{2 J, \ell}\left(r_{<}, r_{>}\right)\right|^{2} r_{<}^{2} r_{>}^{2} d r_{<} d r_{>}=0
$$

Proof. As for the previous result, we have $R_{2 J}\left(r_{<}, r_{>}, r_{12}\right)=h_{2 J}^{2 J+1}\left(r_{<}, r_{>}, r_{12}\right)$ and so using (6.74) with $m=2 J$ and $n=2 J+1$ shows that (6.82) and (6.83) are stronger than the assumption on the right-hand side of (6.52) and the assumption of (6.53), respectively.

Furthermore, Lemma 6.19 removes the need for the assumption on the boundedness of $\left|\mathcal{L}_{j} R_{2 J}\left(r_{<}, r_{>}, r_{12}\right)\right|$ in Lemma 6.13 . The continuity assumption in Lemma 6.13 is shown to be true by the explicit forms derived above.

Hence all assumptions in Lemma 6.13 are satisfied and the result holds.

We are now in a position to prove Theorems 4.2 and 4.3 .

Proof of Theorem 4.2. The expansion of $\psi_{\ell}\left(r_{<}, r_{>}\right)$given in (4.1) follows from (5.1) for $J \rightarrow 2 J$. The decay rates of the norms given in (4.2) follow from the bound (6.42) in Corollary 6.10 with $g=A\left(r_{<}, r_{>}\right)$. Finally the results on the decay of the remainder in (4.3) and (4.4) follow directly from Corollary 6.20.

Proof of Theorem 4.3. The expansion of $\frac{\partial}{\partial r_{\gamma}} \psi_{\ell}\left(r_{<}, r_{>}\right)$given in (4.5) follows directly from (5.1) for $J \rightarrow 2 J+1$. The decay rates of the norms given in (4.6) follow from the bound (6.43) in Corollary 6.10 with $g=A\left(r_{<}, r_{>}\right)$. Finally the results on the decay of the remainder in (4.7) and (4.8) follow directly from Corollary 6.21.

7. Open problems. The obvious extension to this work would be to mathematically prove the required regularity of the helium ground state wavefunction. As discussed in section 3 , this seems to be a formidable task.

However, there are a number of obvious generalizations of this work which would be of interest. Firstly is the extension to other symmetries of two-electron atoms, i.e., nonspherically symmetric cases. Although this has been investigated for the perturbation theoretic case [15], it seems highly nontrivial to extend the current method to such cases. Another interesting case is that of the relativistic atomic Hamiltonian, 
for which there is evidence [20] that the relativistic corrections have partial wave increments $(\ell+1 / 2)^{-2}$ rather than $(\ell+1 / 2)^{-4}$ for the nonrelativistic terms.

A related topic of interest is the rate of convergence with respect to the radial basis. The cases of a Laguerre basis and the natural orbital basis for a CI calculation of the $\ell=0$ part of the ground state of helium have been investigated numerically [17]. A similar rigorous analysis of these asymptotics could lead to insight into the important terms of the expansion, as well as providing rigorous extrapolation formulas.

Finally, the ultimate aim would be to extend such results to more general atoms, and, indeed, molecules, but the route by which this is possible seems unclear at present.

Acknowledgments. This work forms part of a Ph.D. thesis under the supervision of Gero Friesecke, whom I thank for Lemma 4.1, as well as many helpful discussions.

\section{REFERENCES}

[1] M. Abramowitz And I. A. Stegun, Handbook of mathematical functions with formulas, graphs, and mathematical tables, National Bureau of Standards Applied Mathematics Series; 55, U.S. Dept. of Commerce, Washington, D.C., 1972.

[2] M. Born and R. Oppenheimer, Zur Quantentheorie der Molekeln, Ann. Phys., 389 (1927), pp. $457-484$.

[3] M. W. J. Bromley And J. Mitroy, Convergence of the partial wave expansion of the He ground state, Internat. J. Quantum Chem., 107 (2007), pp. 1150-1161.

[4] F. W. Byron And C. J. Johchain, Correlation effects in atoms. II. Angular correlations between electrons, Phys. Rev., 157 (1967), pp. 1-6.

[5] D. P. Carroll, H. J. Silverstone, and R. M. Metzger, Piecewise polynomial configuration interaction natural orbital study of $1 s^{2}$ helium, J. Chem. Phys., 71 (1979), pp. 4142-4163.

[6] V. Fock, Näherungsmethoden zur Lösung des quantenmechanischen Mehrkörperproblems, Z. Phys., 61 (1930), pp. 126-148.

[7] S. Fournais, M. Hoffmann-Ostenhof, T. Hoffmann-Ostenhof, and T. Ø. Sørensen, Sharp regularity results for Coulombic many-electron wave functions, Comm. Math. Phys., 255 (2005), pp. 183-227.

[8] G. FRIESECKE, to appear.

[9] R. GDanitz, Accurately solving the electronic Schrödinger equation of atoms and molecules using explicitly correlated $\left(r_{12}-\right) M R$-CI. II. Ground-state energies of first-row atoms and positive ions, J. Chem. Phys., 109 (1998), pp. 9795-9801.

[10] D. R. Hartree, The wave mechanics of an atom with a non-Coulomb central field, Proc. Cambridge Philos. Soc., 24 (1928), pp. 89-132.

[11] R. N. HiLL, Rates of convergence and error estimation formulas for the Rayleigh-Ritz variational method, J. Chem. Phys., 83 (1985), pp. 1173-1196.

[12] T. Kato, On the eigenfunctions of many-particle systems in quantum mechanics, Comm. Pure Appl. Math., 10 (1957), pp. 151-177.

[13] B. Klahn And W. A. Bingel, The convergence of the Rayleigh-Ritz method in quantum chemistry, Theor. Chim. Acta, 44 (1977), pp. 27-43.

[14] B. Klahn and J. D. Morgan, Rates of convergence of variational calculations and of expectation values, J. Chem. Phys., 81 (1984), pp. 410-433.

[15] W. Kutzelnigg and J. D. Morgan, Rates of convergence of the partial-wave expansions of atomic correlation energies, J. Chem. Phys., 96 (1992), pp. 4484-4508.

[16] W. Lakin, On singularities in eigenfunctions, J. Chem. Phys., 43 (1965), pp. 2954-2956.

[17] J. Mitroy, M. W. J. Bromley, and K. Ratnavelu, Convergence of an s-wave calculation of the He ground state, Internat. J. Quantum Chem., 107 (2007), pp. 907-920.

[18] H. NAKAShima AND H. NAKATSUji, Solving the Schrödinger equation for helium atom and its isoelectronic ions with the free iterative complement interaction (ICI) method, J. Chem. Phys., 127 (2007), p. 224104.

[19] Y. Ralchenko, F.-C. Jou, D. Kelleher, A. Kramida, A. Musgrove, J. Reader, W. Wiese, AND K. OlSen, NIST Atomic Spectra Database (version 3.1.2), National Institute of Standards and Technology, Gaithersburg, MD, 2007. 
[20] S. Salomonson and P. Öster, Relativistic all-order pair functions from a discretized singleparticle Dirac Hamiltonian, Phys. Rev. A, 40 (1989), pp. 5548-5558.

[21] H. M. Schmidt And H. V. HirschHausen, Perturbation theory in $1 / z$ for atoms: First-order pair functions in an l-separated hylleraas basis set, Phys. Rev. A, 28 (1983), pp. 3179-3183.

[22] E. Schrödinger, Quantisierung als Eigenwertproblem, Ann. Phys., 79 (1926), p. 361.

[23] C. Schwartz, Importance of angular correlations betweeen atomic electrons, Phys. Rev., 126 (1962), pp. 1015-1019.

[24] A. Szabo And N. S. Ostlund, Modern Quantum Chemistry, Dover Publications, New York, 1996. 